\title{
BROOKHFWEN
}

NATIONAL LABORATORY

BNL-108214-2015-JA

\section{INVESTIGATION OF REFRACTORY BLACK CARBON-CONTAINING PARTICLE MORPHOLOGIES USING THE SINGLE-PARTICLE SOOT PHOTOMETER (SP2)}

\begin{abstract}
Sedlacek III, A. J., Lewis, E. R., Onasch, T. B., Lambe, A. T., and Davidovits, P. Accepted for publication in Aerosol Science \& Technology
\end{abstract}

July 2015

Biological, Environmental \& Climate Sciences Dept. Brookhaven National Laboratory

\section{U.S. Department of Energy Office of Science}

Notice: This manuscript has been authored by employees of Brookhaven Science Associates, LLC under Contract No. DE-SC0012704 with the U.S. Department of Energy. The publisher by accepting the manuscript for publication acknowledges that the United States Government retains a non-exclusive, paid-up, irrevocable, world-wide license to publish or reproduce the published form of this manuscript, or allow others to do so, for United States Government purposes. 


\section{DISCLAIMER}

This report was prepared as an account of work sponsored by an agency of the United States Government. Neither the United States Government nor any agency thereof, nor any of their employees, nor any of their contractors, subcontractors, or their employees, makes any warranty, express or implied, or assumes any legal liability or responsibility for the accuracy, completeness, or any third party's use or the results of such use of any information, apparatus, product, or process disclosed, or represents that its use would not infringe privately owned rights. Reference herein to any specific commercial product, process, or service by trade name, trademark, manufacturer, or otherwise, does not necessarily constitute or imply its endorsement, recommendation, or favoring by the United States Government or any agency thereof or its contractors or subcontractors. The views and opinions of authors expressed herein do not necessarily state or reflect those of the United States Government or any agency thereof. 
1 Investigation of Refractory Black Carbon-Containing Particle Morphologies using the

2 Single-Particle Soot Photometer (SP2)

3 Arthur J. Sedlacek, III ${ }^{1}$, Ernie R. Lewis ${ }^{1}$, Timothy B. Onasch ${ }^{2,3}$, Andrew T. Lambe ${ }^{2,3}$, and Paul

4 Davidovits $^{3}$

$5{ }^{1}$ Biological, Environmental \& Climate Sciences Department, Brookhaven National Laboratory, 6 Upton, NY 11973

$7 \quad 2$ Aerodyne Research Inc., Billerica, MA 01821

$8 \quad{ }^{3}$ Department of Chemistry, Boston College, Chestnut Hill, MA 02467 
Abstract

11 An important source of the uncertainty in radiative forcing by absorbing aerosol particles is the

12 uncertainty in their morphologies (i.e., the location of the absorbing substance on/in the particles).

13 To examine the effects of particle morphology on the response of an individual black carbon-

14 containing particle in a Single-Particle Soot Photometer (SP2), a series of experiments was

15 conducted to investigate black carbon-containing particles of known morphology using Regal

16 black (RB), a proxy for collapsed soot, as the light-absorbing substance. Particles were formed

17 by coagulation of RB with either a solid substance (sodium chloride or ammonium sulfate) or a

18 liquid substance (dioctyl sebacate), and by condensation with dioctyl sebacate, the latter

19 experiment forming particles in a core-shell configuration. Each particle type experienced

20 fragmentation (observed as negative lagtimes), and each yielded similar lagtime responses in

21 some instances, confounding attempts to differentiate particle morphology using current SP2

22 lagtime analysis. SP2 operating conditions, specifically laser power and sample flow rate, which

23 in turn affect the particle heating and dissipation rates, play an important role in the behavior of

24 particles in the SP2, including probability of fragmentation. This behavior also depended on the

25 morphology of the particles and on the thermo-chemical properties of the non-RB substance.

26 Although these influences cannot currently be unambiguously separated, the SP2 analysis may

27 still provide useful information on particle mixing states and black carbon particle sources. 
30 Black carbon $(\mathrm{BC})$ is of interest to the climate change community because of its large positive

31 contribution to radiative forcing, being second only to $\mathrm{CO}_{2}$ in this regard (Ramanathan and

32 Carmichael, 2008; Bond et al., 2013). The ability of a BC-containing particle to absorb solar

33 radiation is determined primarily by the amount and optical properties of the BC component, but

34 is also affected by other, non-BC components. These non-BC substances may have been present

35 since particle formation or may have been collected subsequently, either by coagulation with

36 other particles or through condensation of gaseous components. The optical properties of a BC-

37 containing particle are strongly dependent upon particle morphology, i.e., particle shape and the

38 location of the BC component within (or on) the particle. Due to the complex interplay among

39 these various factors, and the changes that they may undergo during the lifetime of the particle, a

40 quantitative understanding of $\mathrm{BC}$ radiative forcing has been difficult to obtain. In particular, one

41 of the issues limiting further understanding has been the capability of obtaining real-time, in situ

42 measurements of the morphology of BC-containing particles.

43 The Single-Particle Soot Photometer (SP2, Droplet Measurement Technologies; Schwarz et al., 44 2006; Moteki and Kondo, 2007; Subramanian et al., 2010) is an instrument that measures, in situ, 45 the time-dependent scattering and incandescence signals produced by individual BC-containing 46 particles as they travel through a continuous-wave laser beam. Any particle traversing the laser 47 beam will scatter light, and the BC component of a BC-containing particle will absorb some of 48 the laser energy until its temperature is raised to the point at which it incandesces (hereafter we 49 adopt the standard terminology of the SP2 community and denote any substance determined by 50 the SP2 to be BC as refractory black carbon $(\mathrm{rBC})$ ). The scattering signal provides information 51 on particle size and the incandescence signal is used for counting rBC-containing particles and 52 yields the mass of the $\mathrm{rBC}$ component within each particle (obtained via calibration). Hence, the 53 SP2 can determine size-resolved number and mass concentrations of rBC-containing particles 54 and thus provide information on the rBC particle population-based mixing states within ambient 55 aerosols. In addition, SP2 scattering and incandescence signals have recently been used to 56 estimate the amount of the non-rBC component and infer the structure of individual rBC57 containing particles, therefore suggesting the possibility that this instrument can also provide 58 information on the morphology of rBC-containing particles (Sedlacek et al., 2012). 
59 The amount of non-rBC material associated with an $\mathrm{rBC}$-containing particle can be estimated by

60 two different methods, both of which assume a core-shell configuration in which a spherical rBC

61 core is surrounded by a uniform concentric shell of non-rBC material and require assumptions

62 regarding the densities and refractive indices of the $\mathrm{rBC}$ and non-rBC substances comprising the

63 particle. The "leading edge optimization (LEO)" method (Gao et al., 2007; Schwarz et al., 2008;

64 Moteki and Kondo, 2008) is based on the temporal behavior of the scattering signal and on the

65 peak magnitudes of the scattering and incandescence signals. The scattering signal will initially

66 increase as the particle enters the laser beam, exhibiting a Gaussian dependence with time

67 (following the spatial profile of the laser intensity, which the particle transits at constant speed).

68 The magnitude of this signal will attain a maximum value and eventually decrease as the particle

69 becomes smaller due to evaporation of the non-rBC substance, the energy absorbed by the rBC

70 being dissipated as latent heat of this non-rBC material. The initial portion of the time-dependent

71 scattering signal (i.e., before any appreciable evaporation of the non-rBC material has occurred)

72 is fitted to a Gaussian, yielding an estimate of the original optical diameter of the particle. This

73 quantity, together with the $\mathrm{rBC}$ mass (which is obtained from the peak magnitude of the

74 incandescence signal), can be used to determine the mass of the non-rBC material. Under the

75 core-shell assumption, the amount of the non-rBC material can also be quantified as a coating 76 thickness.

77 The "lagtime" method (Moteki and Kondo, 2007; Subramanian et al., 2010) employs the 78 temporal behaviors of both the scattering and incandescence signals. The lagtime $(\Delta \tau)$ is defined 79 as the difference in the time of the peak in the (abrupt) incandescence signal, which occurs when 80 all non-rBC material has been removed and the temperature of the rBC has risen to the point 81 where it incandesces, and the time of the maximum value of the scattering signal. This quantity 82 provides a proxy for the amount of non-rBC material - a greater lagtime indicating a larger 83 amount of substance that must evaporate before the remaining rBC can incandesce (Schwarz et

84 al., 2006; Moteki and Kondo 2007). This approach has been used to categorize rBC-containing 85 particles as either aged or fresh, the two extremes relating to the amount of time since production 86 of the particle and thus the amount of condensation of non-refractory material that has occurred 87 (e.g., Subramanian et al., 2010). 
It had been typically assumed that the SP2 lagtime should always be greater than or equal to zero

89 for a core-shell configuration, as any non-rBC substance must evaporate before the rBC can 90 attain a sufficient temperature to incandesce (and sublimate), after which no material remains to 91 produce any scattering signal. However, Moteki and Kondo (2007) observed negative lagtimes 92 (i.e., the maximum value of the scattering signal occurring after incandescence) in a laboratory 93 study involving rBC particles known to be in a core-shell configuration (having been formed by 94 condensation of gases on a nearly spherical soot core) with a large amount of non-refractory 95 material. They hypothesized that these negative lagtimes resulted from particle fracturing when 96 the rBC could not dissipate the absorbed heat sufficiently rapidly to the non-rBC substance. This 97 fracture would allow some of the remaining non-rBC material to pass through the laser and 98 produce a scattering signal after the rBC had incandesced.

99 Sedlacek et al. (2012) observed negative lagtimes for a large fraction of ambient rBC-containing 100 particles in some air masses. However, some of these particles, unlike those in the previous 101 laboratory measurements, did not appear (based on the optical diameter method) to have large 102 amounts of non-rBC material. These investigators concluded that the negative lagtimes occurred 103 for particles for which the $\mathrm{rBC}$ component was at or near the surface. In particular, biomass 104 burning plumes were found to contain high fractions of such particles in several field studies, 105 suggesting that this fraction could be used for source attribution. Other investigations (Dahlkötter 106 et al., 2014; Moteki et al., 2014) have subsequently reported similar observations; for example, 107 Moteki et al. (2014), reported that the fraction of ambient BC-containing particles in Tokyo with 108 the $\mathrm{rBC}$ near the surface of the particle was typically less than $10 \%$ but increased to near $40 \%$ in 109 some cases. The conclusions that these observations result from BC being at the surface of the 110 particles, and which are based on the temporal behavior of the scattering and incandescence 111 signals observed for apparently different rBC-containing particle morphologies, may have 112 important implications on the global radiative forcing by BC-containing particles, especially 113 those from biomass burning.

114 In their work, Moteki et al. (2014) described a third approach; a new SP2 mixing analysis 115 methodology that utilizes the behavior of the time-dependent scattering cross-section to classify 116 the particle morphology type. This method uses aspects from both the LEO and the lagtime 117 approaches and employs three quantities: the measured scattering cross-section of the particle 
118 prior to evaporation (i.e., as it first enters the laser beam), the measured scattering cross-section

119 at the start of incandescence, and an independently calibrated scattering cross-section of 120 uncoated $\mathrm{rBC}$. The ratio of the first two quantities provides information on particle evaporation;

121 a ratio near unity implies that there is limited thermal contact between the absorbing rBC 122 component and non-refractory component and thus limited evaporation (i.e., what they refer to as 123 "attached type"), whereas at the other extreme, a ratio much less than unity implies that there is 124 greater thermal contact, such as what would be expected for a core-shell configuration (i.e., what 125 they refer to as "coated type"). The ratio of the scattering cross-section of the particle prior to 126 evaporation to the scattering cross-section of the $\mathrm{rBC}$ alone quantifies the amount of non-rBC 127 material and is used to define a range of conditions where particle morphology can be inferred 128 from the first ratio. Direct comparisons between this new methodology and the lagtime approach 129 have not yet been conducted; however, both methods provide mechanisms for inferring particle 130 morphology based on the time-depedent behavior of the light scattered by rBC-containing 131 particles relative to the $\mathrm{rBC}$ incandescence signal.

132 The current manuscript explores the utility of the lagtime method to infer particle morphology by 133 conducting a systematic series of laboratory experiments to measure the SP2 scattering and 134 incandescence signals from rBC-containing particles with different morphologies and chemical 135 compositions. Specifically, laboratory particles were generated containing rBC and either an 136 inorganic salt or an organic substance, and with the rBC either on the surface, via coagulation, or 137 as the core of a core-shell configuration, via condensation. Additionally, the SP2 scattering and 138 incandescence signals measured from particles of these types were investigated as a function of 139 the SP2 operating conditions, i.e., laser power and sample flow rate.

\section{Experimental Details}

141 In all experiments, atomized Regal black (RB, Cabot Corp), which has a compact, aciniform 142 solid structure, was used for the rBC component and was chosen as a surrogate for collapsed soot. 143 Scattering and incandescence signals from individual particles were measured with an 8-channel 144 SP2 (revision D). The mass of the rBC contained in a particle is determined from the peak 145 magnitude of the incandescence signal by calibration with Regal black, and is reported as a mass-equivalent diameter $\left(D_{\mathrm{me}, \mathrm{rBC}}\right)$ based on an assumed density of $1.8 \mathrm{~g} \mathrm{~cm}^{-3}$. The SP2 can 
147 detect incandescence signals from particles with $D_{\text {me,rBC }}$ in the range $\sim 70-600 \mathrm{~nm}$, but scattering 148 signals only from particles with initial diameters in the range $\sim 175-350 \mathrm{~nm}$.

149 Particle number concentrations were measured with a condensation particle counter (CPC), and 150 particle mobility distributions were measured with a scanning mobility particle sizer (SMPS). 151 Sample preparation conditions (i.e., solute concentrations, etc.) were chosen to maximize the 152 number of particles with sizes within the detection range of the SP2 scattering channel to 153 facilitate utilization of the lagtime technique.

154 In the first set of experiments, lagtimes were determined from SP2 scattering and incandescence 155 signals from particles formed by coagulation of $\mathrm{RB}$ with sodium chloride $(\mathrm{NaCl}$; hereafter 156 referred to as $\mathrm{SC})$ and by coagulation of $\mathrm{RB}$ with ammonium sulfate $\left(\left(\mathrm{NH}_{4}\right)_{2} \mathrm{SO}_{4}\right.$; hereafter 157 referred to as $\mathrm{AS}$ ), forming $\mathrm{RB}+\mathrm{SC}$ and $\mathrm{RB}+\mathrm{AS}$ particles, respectively. As both of these particle 158 types are aggregates composed of two solid substances, they clearly cannot possess core-shell 159 configurations. Both SC and AS were used because these substances have different thermo160 chemical properties such as melting point, thermal diffusivity, specific heat, vapor pressure, and 161 latent heat, and differences in these quantities would be expected to affect the rate and amount of 162 intra-particle heat transfer and thus the lagtime behavior. For example, the melting point of AS $163\left(235^{\circ} \mathrm{C}\right)$ is much lower than that of SC $\left(800{ }^{\circ} \mathrm{C}\right)$, and its specific heat at $300 \mathrm{~K}\left(188 \mathrm{~J} \mathrm{~mol}^{-1} \mathrm{~K}^{-1}\right)$ 164 is much greater than that of $\mathrm{SC}\left(50 \mathrm{~J} \mathrm{~mol}^{-1} \mathrm{~K}^{-1}\right)$. It should be noted that although in many 165 techniques (such as thermal volatility) SC is considered to be refractory, in the present context it 166 is considered to be a non-refractory substance.

167 The particle generation and sampling systems used for these measurements are shown in 168 Figure 1. RB, SC, and AS were generated separately by atomization from aqueous solutions and

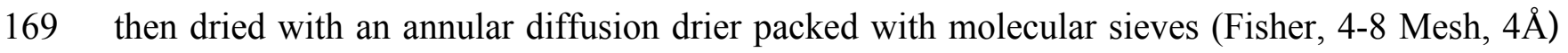
170 to maintain relative humidity conditions below the efflorescence relative humidities of SC and $171 \mathrm{AS}$, near 45\% and 40\%, respectively (Tang et al., 1996). The aerosol consisting only of 172 absorbing and refractory RB particles and the aerosol consisting only of non-absorbing and non173 refractory particles (SC or AS) were initially characterized separately to obtain desired number 174 concentrations and mean sizes, and then these aerosols were combined in a closed stainless steel 175 drum (SKOLNIK, 55 gal) for 0.6 to $20.6 \mathrm{hrs}$ to allow coagulation of rBC and non-rBC particles. 
176 High number concentrations $\left(\sim 10^{5} \mathrm{~cm}^{-3}\right)$ were used to increase coagulation rates. Prior to each 177 experiment, the drum was flushed with particle-free air until the background particle concentration was less than $10 \mathrm{~cm}^{-3}$. The $\mathrm{rBC}$ and non-rBC aerosols were continually introduced 179 into the drum for $\sim 1 \mathrm{hr}$ until number concentrations reached steady-state values. The drum was 180 then isolated at both ends, allowing the particle size distributions to evolve via coagulation, wall 181 loss, and gravitational sedimentation. Aerosol samples were withdrawn from the drum (and the 182 extracted volume was replaced with filtered air) at intervals to examine changes in particle 183 properties as a function of mixing time $\left(t_{\text {mix }}\right)$. A HEPA filter diluter placed downstream of the 184 drum before the SP2 reduced the aerosol number concentration by a factor of $\sim 100$ to minimize 185 particle coincidence during single-particle measurements. Size distributions of $\mathrm{rBC}$ number 186 concentration for particles formed by coagulation of RB and SC measured by the SP2 at various 187 mixing times are shown in Figure 2; the increase in $D_{\text {me,rBC }}$ with increasing time demonstrating 188 that $\mathrm{RB}+\mathrm{RB}$ coagulation occurred and thus confirming that the experimental conditions were 189 conducive to coagulation.

190 In another set of experiments, RB particles were (a) coagulated with drops of dioctyl sebacate 191 (DOS, an organic substance that is liquid at room temperature) generated via homogenous 192 nucleation in a heated flask, and (b) coated with DOS via gas-to-particle condensation, forming $193 \mathrm{RB}+\mathrm{DOS}$ and $\mathrm{DOS}=>\mathrm{RB}$ particles, respectively. In the coagulation experiments, the techniques 194 and sampling were the same as those used in the previous coagulation experiments with RB and 195 SC or AS. In the condensation experiments, the DOS $=>$ RB particles were formed by passing RB 196 particles through a chamber with a heated bath containing DOS at various temperatures (103, 197107 , and $115^{\circ} \mathrm{C}$ ), the dependence of vapor pressure on temperature resulting in different 198 concentrations of DOS vapor and thus different coating thicknesses. The DOS=>RB particles 199 were sampled directly after leaving the chamber. This set of experiments was conducted to 200 investigate the SP2 responses to particles formed by two different methods that may (at least 201 initially) have different morphologies; i.e., $\mathrm{DOS}=>\mathrm{RB}$ particles are expected to have a core-shell 202 configuration, whereas RB+DOS particles will not, at least initially. However, RB may become 203 wetted by the liquid DOS and the coagulated particles may change morphologies (e.g., become 204 more core-shell like) over time. 
205 A final set of experiments investigated the dependence of the lagtimes on SP2 operating 206 conditions, specifically laser power (qualitatively indicated by laser diode current) and sample 207 flow rate, both of which affect the heating rate of rBC-containing particles. Increasing the pump 208 laser power results in increased intracavity laser intensity, and increasing the sample flow rate 209 results in increased particle velocity, both of which lead to a greater rate of energy absorption by 210 the $\mathrm{rBC}$, less time for intra-particle heat transfer from the $\mathrm{rBC}$ to the non-rBC material, and thus 211 increased particle heating rate. To examine the relationship between particle velocity and flow 212 rate, the full width at half maximum (FWHM) of the scattering signals from purely scattering $213 \mathrm{NaCl}$ particles was measured at different sample flow rates. As the width of the Gaussian laser 214 beam is independent of the sample flow rate, the inverse of the FWHM is directly proportional to 215 the particle velocity, which from Figure 3 is seen to increase linearly with sample flow rate. 216 Two types of experiments were performed. In the first, the sample flow rate was held constant 217 and the SP2 laser power was varied by changing the injection current of the pump laser over a 218 range of a factor of 1.5, from 2000 and $3000 \mathrm{~mA}$. In the second, the laser power was held 219 constant and the sample flow rate was varied over a factor of 4 from 82 to $360 \mathrm{~cm}^{3} \mathrm{~min}^{-1}$ by 220 changing the exhaust flow rate while leaving the sheath flow rate constant. All other experiments 221 described in this manuscript were performed with the laser power at $2500 \mathrm{~mA}$ and the sample 222 flow rate at $120 \mathrm{~cm}^{3} \mathrm{~min}^{-1}$.

\section{Results}

225 Single particle SP2 lagtime measurements. Several types of single-particle SP2 scattering and 226 incandescence signals were observed during this study, with varying frequency depending on the 227 particle system studied. Examples for $\mathrm{RB}+\mathrm{SC}$ and $\mathrm{RB}+\mathrm{DOS}$ particles are shown in Figure 4a-e 228 and Figure 4f-g, respectively. In the first type (Figure 4a), the maximum of the scattering signal occurs before that of the incandescence signal, resulting in a positive lagtime. Under the core230 shell assumption, this particle would be interpreted as having an $\mathrm{rBC}$ core with sufficient 231 associated non-rBC material that it requires an appreciable time to absorb enough laser energy to 232 evaporate this material. In the second type (Figure 4b), the maxima of the scattering and 233 incandescence signals occur at nearly the same time, resulting in a lagtime near zero. Such 234 signals are interpreted as resulting from $\mathrm{rBC}$-containing particles with little or no non-rBC 
235 material. In the third type (Figure 4c), the maximum of the scattering signal occurs after the peak 236 of the incandescence signal and thus results in a negative lagtime, behavior seemingly 237 inconsistent with the conventional core-shell assumption. SP2 signals with two scattering peaks 238 were also observed; for some of these (Figure 4d) the largest peak occurred before incandescence, 239 whereas for others (Figure 4e) it occurred after incandescence. As the lagtime is defined as the 240 time difference between the peak of the incandescence signal and the maximum value of the 241 scattering signal (regardless of any other features of the scattering signal), these two situations 242 correspond to positive and negative lagtime, respectively. For RB+DOS particles two scattering 243 peaks were also observed, with the smaller one occurring before incandescence and the larger 244 one occurring either simultaneously with (Figure 4f) or after (Figure 4g) incandescence.

245 Lagtime distribution plots. The distributions of lagtimes (derived from the time-dependent SP2 246 scattering and incandescence signals from individual particles) from each set of coagulation 247 experiments $(\mathrm{RB}+\mathrm{SC}, \mathrm{RB}+\mathrm{AS}$, and $\mathrm{RB}+\mathrm{DOS})$ for short $(<1 \mathrm{hr})$, intermediate $(1.5-2 \mathrm{hr})$, and 248 long $(>17 \mathrm{hr})$ mixing times are plotted in Figure 5 as a function of $\mathrm{rBC}$ mass-equivalent 249 diameter $D_{\text {me,rBC. }}$. Colors represent the number fraction of particles within given ranges of $\Delta \tau$ 250 (400 ns) and $D_{\mathrm{me}, \mathrm{rBC}}(5 \mathrm{~nm})$. In each case, the normalized size distribution of RB-number 251 concentration in the representation $d N_{\mathrm{rBC}} / d \log D_{\mathrm{me}, \mathrm{rBC}}$ is shown, as is the ratio of the number of 252 particles with positive lagtimes to those with negative lagtimes (positive-to-negative lagtime 253 ratio). Since positive and negative lagtimes only have meaning for $\mathrm{rBC}$ particles containing 254 associated non-rBC material, this ratio excludes uncoated $\mathrm{rBC}$ particles. As the majority of RB 255 particles remained uncoagulated with SC, AS, or DOS even at the longest mixing time, lagtimes 256 of these particles are not included in the lagtime distribution figures. An RB particle was 257 assumed to be uncoagulated if it had a value of $\Delta \tau$ in the range -0.4 to $1.2 \mu \mathrm{s}$; this upper limit, 258 though somewhat arbitrary, is commonly accepted in the SP2 research community and allows for 259 uncertainties in instrument response. As particles resulting from coagulation between two or 260 more RB particles will fall into this range, the lagtimes distributions presented thus pertain only 261 to particles resulting from coagulation of $\mathrm{RB}$ and the non-rBC substance.

262 Coagulation of Regal Black (RB) and Sodium Chloride (SC). Lagtime distributions for RB+SC 263 particles are shown in the left column of Figure 5 as a function of $D_{\mathrm{me}, \mathrm{BBC}}$ for mixing times 264 ranging from $0.6 \mathrm{hr}$ up to $17 \mathrm{hr}$. All these distributions are qualitatively similar and have a 
265 prominent mode at small positive lagtimes (near $\sim 1.5 \mu \mathrm{s}$ ) that extends to larger positive lagtimes

266 at smaller values of $D_{\text {me,rBC}}$, and another mode at negative lagtimes that covers a wider range of 267 values of $\Delta \tau$ (from -1 to $-3 \mu \mathrm{s}$ ). The location of the maximum value of $D_{\text {me,rBC }}$ for the mode at 268 small positive lagtimes increases with increasing mixing time as a result of coagulation and 269 gravitational settling processes. The most striking observation for the $\mathrm{RB}+\mathrm{SC}$ system is the 270 presence of both negative and positive lagtimes, which occur in roughly equal numbers (a 271 positive-to-negative lagtime ratio of 0.7 implies that $41 \%$ of the lagtimes are positive, and a $+/-$ 272 ratio of 1.2 implies that $54 \%$ of the lagtimes are positive). The occurrence of negative lagtimes is 273 consistent with the original interpretation put forth by Sedlacek et al. (2012) that they originate 274 from the $\mathrm{rBC}$ being located at or near the surface, but the presence of positive lagtimes is 275 unexpected for the current situation of coagulated solid-solid particles. Possible reasons for these 276 positive lagtimes will be discussed below.

277 Coagulation of Regal Black (RB) and Ammonium Sulfate (AS). Lagtime distributions for RB+AS 278 particles for mixing times of $1 \mathrm{hr}$ and $20.6 \mathrm{hr}$ are shown in Figure 5, middle column. At the 279 shortest mixing times, there are fewer $\mathrm{RB}+\mathrm{AS}$ coagulated particles than for $\mathrm{RB}+\mathrm{SC}$ at the 280 shortest mixing time (probably because of differences in the relative distributions of SC, AS, and $281 \mathrm{RB}$ for the different situations), and no positive-to-negative lagtime ratio is presented for this 282 situation. At the longest mixing time, the RB+AS lagtime distribution is qualitatively similar to 283 that for $\mathrm{RB}+\mathrm{SC}$ at all mixing times and exhibits both positive and negative lagtimes, although 284 the fraction of negative lagtimes for RB+AS particles is substantially smaller than that for $285 \mathrm{RB}+\mathrm{SC}$ particles (the positive-to-negative lagtime ratio is 12.5 for $\mathrm{RB}+\mathrm{AS}$ compared to 1.2 for $286 \mathrm{RB}+\mathrm{SC})$.

287 Coagulation of Regal Black (RB) and Dioctyl Sebacate (DOS). Lagtime distributions for 288 RB+DOS particles (Figure 5, right column) also exhibit both negative and positive lagtimes. The 289 distribution for the shortest mixing time is qualitatively similar to those for the RB+SC system at 290 all mixing times (and to that for RB+AS at the longest mixing time); specifically, there is a 291 dominant mode at positive lagtimes centered near $1.5 \mu$ s distributed over a range of values of $292 D_{\text {me,rBC }}$ and another, less prominent mode at negative lagtimes distributed over a wide range of 293 lagtimes. In contrast to the $\mathrm{RB}+\mathrm{SC}$ and $\mathrm{RB}+\mathrm{AS}$ cases, however, the $\mathrm{RB}+\mathrm{DOS}$ distribution at the 294 longest mixing time looks qualitatively different and has a second negative lagtime mode 
centered near $-3.4 \mu \mathrm{s}$ and $150 \mathrm{~nm}$. The RB+DOS lagtime distributions exhibit a greater change with increasing mixing time than do those for $\mathrm{RB}+\mathrm{SC}$ or $\mathrm{RB}+\mathrm{AS}$; the secondary positive lagtime mode at small $D_{\text {me,rBC }}$ values disappears, as does the primary, small magnitude negative lagtime mode.

299 Scattering signals for individual particles also exhibit significant differences between the $\mathrm{RB}+\mathrm{DOS}$ and $\mathrm{RB}+\mathrm{SC}$ cases. For the shortest mixing times both $\mathrm{RB}+\mathrm{DOS}$ and $\mathrm{RB}+\mathrm{SC}$ particles with positive lagtimes exhibit scattering signals similar to that shown in Figure 4a, but for the

302 longest mixing time the scattering signals from RB+DOS particles exhibit two peaks, either one 303 before incandescence and one coincident with incandescence (Figure 4f), or one before 304 incandescence and one after (Figure 4g), whereas those from RB+SC particles exhibit only one 305 peak. This will be discussed further below.

Condensation of DOS on Regal Black $(R B)$. Lagtime distributions for DOS $=>\mathrm{RB}$ particles are shown in Figure 6 (middle column) as a function of DOS bath temperature. As the RB size distribution was the same for all bath experiments, $d N_{\mathrm{rBC}} / d \log D_{\mathrm{me}, \mathrm{rBC}}$ is shown in only the top panel. Additionally, as there were no uncoated RB particles in this study, signals with small lagtimes were not removed from the graphs and analysis, as they were for the coagulation studies.

311 For the sizes of particles and the parameters characterizing the condensation, to good 312 approximation the thickness of the DOS coating would be independent of initial RB diameter 313 and directly proportional to the vapor pressure of DOS, which (based on values given in $314 \mathrm{O}^{\prime}$ Hanlon, 2003) increases by a factor of $\sim 1.4$ between $103^{\circ} \mathrm{C}$ and $107^{\circ} \mathrm{C}$, and a factor of $\sim 2$ 315 between $107^{\circ} \mathrm{C}$ and $115^{\circ} \mathrm{C}$. However, temperature inhomogeneities in the chamber will result in 316 some spread in the DOS coating thicknesses at any nominal temperature.

317 DOS $=>$ RB particles exhibit both positive and negative lagtimes (Figure 6), but also lagtimes 318 near zero, despite the fact that all particles in these condensation experiments were coated and 319 presumably in a core-shell configuration. The lagtime distributions for the $\mathrm{DOS}=>\mathrm{RB}$ particles at 320 the two lower bath temperatures are qualitatively similar to those for RB+DOS at the longest 321 mixing time, but none of the $\mathrm{DOS}=>\mathrm{RB}$ lagtime distributions are similar to any of those for $322 \mathrm{RB}+\mathrm{SC}$ or $\mathrm{RB}+\mathrm{AS}$ particles, or for those for $\mathrm{RB}+\mathrm{DOS}$ particles at the shortest mixing times. 323 Specifically, $\mathrm{DOS}=>\mathrm{RB}$ negative lagtime distributions do not exhibit the small lagtime mode 
324 (lagtimes near $1 \mu$ s and $D_{\mathrm{me}, \mathrm{rBC}}$ between 200 and $300 \mathrm{~nm}$ ) observed for these other particles. The 325 DOS $=>$ RB lagtime distributions contain two distinct modes for all bath temperatures, one at 326 positive values of $\Delta \tau$ and one at negative values. With increasing DOS coating thickness

327 (increasing bath temperature), there is a large shift in the distribution from the positive mode to 328 the negative mode (positive-to-negative lagtime ratio decreasing from 3.4 at $103^{\circ} \mathrm{C}$ to 0.3 at $329115^{\circ} \mathrm{C}$ ), with the lagtimes changing from positive to negative without taking intermediate values. 330 A similar increase in negative lagtimes with increasing coating thickness was reported by Moteki 331 and Kondo (2007).

332 Dependence of Lagtime Distributions on SP2 Operating Conditions. Lagtime distributions for $333 \mathrm{RB}+\mathrm{SC}$ particles in which laser power and sample flow rate were varied are shown in Figure 7, 334 with laser power increasing from top (2000 mA) to bottom (3000 mA) and sample flow rate 335 increasing from left to right $\left(82,120\right.$, and $\left.240 \mathrm{~cm}^{3} \mathrm{~min}^{-1}\right)$. At the lowest laser power and sample 336 flow rate considered $\left(2000 \mathrm{~mA}\right.$ and $\left.82 \mathrm{~cm}^{3} \mathrm{~min}^{-1}\right)$, the lagtimes are predominantly positive 337 (positive-to-negative lagtime ratio 6.1), behavior that would be typically ascribed to a core-shell 338 configuration (Figure 7a). With increasing sample flow rate at this same laser power (Figure 7b339 c), the shapes of the lagtime distributions at positive lagtimes remain qualitatively similar, but a 340 mode begins to appear at negative lagtimes concomitant with a decrease in the positive-to341 negative lagtime ratio. Likewise, with increasing sample flow rate at laser power $3000 \mathrm{~mA}$, the 342 shapes of the lagtime distributions at positive lagtimes are qualitatively similar to each other 343 (Figure 4d-f), with a maximum remaining unchanged near $D_{\mathrm{me}, \mathrm{rBC}}=290 \mathrm{~nm}$, but there is an 344 increase in the frequency of occurrence of negative lagtimes, which eventually dominate over 345 positive lagtimes. At a given sample flow rate, the lagtime distributions at higher laser power 346 (3000 mA) are qualitatively similar to those at lower laser power $(2000 \mathrm{~mA})$, but exhibit large 347 increases in the number of particles with negative lagtimes (and correspondingly large decreases 348 in the positive-to-negative lagtime ratios). The mean values of the negative lagtimes are also 349 larger.

350 The results of these experiments are also summarized in Figure 8, which encompasses a wider 351 array of conditions that those shown in Figure 7. There is a systematic decrease in the positive352 to-negative lagtime ratio with either increased laser power or increased sample flow rate, both of 353 which increase particle heating rate. Over the range of SP2 laser powers and sample flow rates 
354 examined, the laser power exerts a much larger influence on the lagtime distributions than does 355 the sample flow rate.

356 Lagtime distributions for $\mathrm{DOS}=>\mathrm{RB}$ particles under different laser powers and sample flow rates,

357 but at the same bath temperature (and thus coating thickness) are shown in the bottom row of

358 Figure 6. With increased particle heating rate (i.e., left to right), the positive lagtime mode 359 essentially disappears and is replaced by a negative lagtime mode, and the positive-to-negative 360 lagtime ratio decreases from 1.6 to 0.03 .

\section{Discussion}

362 Single-particle energy balance in the SP2. The behavior of an $\mathrm{rBC}$-containing particle in the SP2 363 is determined both by the rate at which the $\mathrm{rBC}$ absorbs energy from the laser and by the rate at 364 which this energy is dissipated (Moteki and Kondo, 2007). This dissipation occurs primarily 365 through conduction to the non-rBC material, from which the energy is subsequently removed 366 through heating and evaporation (i.e., latent heat) of this substance and through conduction to the 367 surrounding gas. The rates of energy absorption and dissipation depend on several factors, the 368 most fundamental of which are the masses and configuration of the $\mathrm{rBC}$ and non-rBC components (e.g., core-shell vs. on surface). These determine the absorption cross-section and 370 thus the rate at which the particle absorbs incident radiation, and they also govern the rate of 371 intra-particle heat dissipation and the rate of heat conduction to the surrounding gas. This 372 dissipation can be so rapid for particles with a sufficiently small BC mass that these particles 373 never incandesce, and thus will not be detected by the SP2. This effect explains the small particle 374 size limit for SP2 incandescence detection (Schwarz et al., 2010), and it also explains the 375 increased number of lagtimes observed for particles with smaller $D_{\mathrm{me}, \mathrm{rBC}}$ under higher laser 376 power condition (Figure 7, bottom row).

377 The primary factors that affect the rate at which a given $\mathrm{rBC}$-containing particle absorbs energy 378 are the SP2 operating conditions, specifically the laser power, which determines the light 379 intensity at a given location, and the sample flow rate, which governs the speed of the rBC 380 particles through the laser. The effects of these instrument operating conditions on the positive381 to-negative lagtime ratio are shown in Figures 7 and 8. Under low laser power and low sample 382 flow rate, the rate at which energy is absorbed by the $\mathrm{rBC}$ is relatively slow, and much of the 
energy can be transferred to and through the non-absorbing, non-refractory substance, 384 evaporating most or all of this substance before the $\mathrm{rBC}$ attains its sublimation temperature, and resulting in a positive lagtime. Conversely, under sufficiently high laser power and sample flow rate, the rate at which energy is absorbed by the $\mathrm{rBC}$ may be so high that this energy cannot be transferred to and through the non-rBC substance before some of this substance at the interface between the non-rBC and $\mathrm{rBC}$ evaporates. Depending on the extent of this evaporation and on the morphology of the particle, the non-rBC and $\mathrm{rBC}$ components can separate, with some of the separated non-rBC material continuing through the SP2 laser as a pure scatterer. Such a situation could result in either a positive or negative lagtime (e.g., Figure 4d-e), depending on the amount 392 of this remaining material. This mechanism was proposed by Moteki and Kondo (2007) for the observation of negative lagtimes observed for thickly coated $\mathrm{rBC}$-containing particles in a coreshell-like configuration, and might also explain the results shown in Figure 6.

The primary factors that affect the intra-particle heat dissipation rate and the subsequent evaporation of the non-rBC component for a given $\mathrm{rBC}$-containing particle are the thermochemical properties of this non-rBC substance (e.g., thermal diffusivity, specific heat, latent heat, etc.) and the extent of contact between the $\mathrm{rBC}$ and non-rBC substances. Evidence for the importance of the non-rBC thermo-chemical properties is the observed difference in behavior 400 between $\mathrm{RB}+\mathrm{SC}$ and $\mathrm{RB}+\mathrm{AS}$ particles. Despite the fact that both of these systems represent 401 solid-solid coagulation, the latter exhibits a much higher fraction of positive lagtimes (Figure 5), 402 likely reflecting the greater thermal volatility of AS compared to SC. Evidence for the 403 importance of the extent of contact between the $\mathrm{rBC}$ and non-rBC components is provided by 404 comparison of the lagtime distributions for the coagulated RB+DOS particles at the shortest 405 mixing times (Figure 5, upper right panel) and the condensed DOS=>RB particles (Figure 6, top 406 panel, which has the same SP2 operating conditions); the former has a much lower positive-to407 negative ratio, probably due to the particles in this system having much less physical and thus 408 thermal contact between the DOS and RB.

409 It is the balance between these rates of energy absorption and energy dissipation that determines 410 the lagtime for a given rBC-containing particle. As such a particle moves through the SP2 it 411 initially scatters light, but this scattering signal attains a maximum value that is determined by 412 the competing effects of a decreasing scattering cross section resulting from particle evaporation 
413 and the increasing laser light intensity as the particle moves further into the laser beam. If the 414 non-rBC substance evaporates completely, this scattering maxima will occur before (or at) 415 incandescence, resulting in a positive (or zero) lagtime. However, if the non-rBC and rBC 416 components separate, then the separated non-rBC particle (or particles) will pass through the 417 laser beam as a pure scatterer, with its (their) scattering cross section(s) remaining constant. The 418 resulting scattering signals for each of the separated, non-absorbing particles will be Gaussian, 419 following that of the laser intensity, with maxima at times when the particles are at the center of 420 the laser (where the intensity is greatest). In contrast, the initial scattering maximum will be non421 Gaussian. As the lagtime is defined as the difference between the time of the peak of the abrupt 422 incandescence of the $\mathrm{rBC}$ and the time of the largest value of the scattering signal, a separated 423 particle could have either a positive or a negative lagtime, depending on the relative magnitudes 424 of the scattering maxima. Thus, two situations that are qualitatively very similar could be 425 classified as quantitatively very different.

426 Lagtimes of individual particles. This laboratory investigation explicitly demonstrates that both 427 positive and negative lagtimes can result from particles formed by the coagulation of two solid 428 particles, the coagulation of a solid particle and a liquid droplet, and the condensation of liquid 429 material on a solid particle. Thus, attempts to infer particle morphology from lagtimes alone 430 must be undertaken with extreme caution. In particular, interpretations of lagtime observations 431 based on the assumption of core-shell particle configurations must be regarded with considerable 432 suspicion. Additionally, negative lagtimes were observed for each particle type, and accounted 433 for at least $10 \%$ of the $\mathrm{rBC}$ particles sampled for each experiment, from which it must be 434 concluded that such lagtimes do not correspond to anomalies, but are expected for these, and 435 presumably other, types of BC-containing particles. For example, Sedlacek et al. (2012) also 436 observed negative lagtimes for $\sim 10 \%$ of ambient BC-containing particles under background 437 conditions when biomass burning plumes were not present, and similar values were reported by 438 Moteki et al. (2014) for ambient urban particles. Finally, a substantial fraction of particles in 439 each experiment exhibited scattering signals with two maxima, and as discussed above, such a 440 signal may correspond to either a positive or a negative lagtime, depending on the relative 441 magnitudes of these maxima (Figure 4). As also noted above, the magnitudes, and even the sign, 442 of the lagtime are not intrinsic properties of an $\mathrm{rBC}$-containing particle, but depend on the rates 443 of energy absorption and dissipation, and thus on SP2 operating conditions. 
444 Lagtime distributions Two distinct patterns are observed for lagtime distributions in these 445 experiments. The first pattern contains three modes: one with rather small positive values of $\Delta \tau$ 446 extending over a range of values of $D_{\text {me,rBC}}$; a second, smaller, mode at larger positive values of $447 \Delta \tau$ at small values of $D_{\mathrm{me}, \mathrm{rBC}}$; and a third mode extending over a wider range of negative values 448 of $\Delta \tau$ (Figure 5, except lower right panel; Figure 7). This first pattern was observed for $\mathrm{RB}+\mathrm{SC}$ 449 and $\mathrm{RB}+\mathrm{AS}$ particles at all mixing times, and for RB+DOS particles at the shorter two mixing 450 times. The second pattern consists of a mode with positive values of $\Delta \tau$ increasing with 451 decreasing $D_{\text {me,rBC}}$, or a mode with negative values of $\Delta \tau$, the magnitude of which decreases with 452 decreasing $D_{\text {me,rBC }}$ and sometimes extends to positive values of $\Delta \tau$, or both (Figure 6 and the 453 lower right panel of Figure 5). This second pattern was observed for DOS $=>$ RB particles and $454 \mathrm{RB}+\mathrm{DOS}$ particles at the longest mixing times.

455 The similarity between the lagtime distributions observed for the RB+DOS particles at the 456 shortest mixing times (Figure 5, upper two panels in right column) and that for $\mathrm{RB}+\mathrm{SC}$ at all 457 mixing times (best seen in Figure 5 in the lower left panel) suggests that the DOS initially had 458 limited contact with the RB and that the two components could easily separate under the 459 operating conditions of this study. The similarity between the lagtime distributions observed for 460 the RB+DOS particles at the longest mixing time (Figure 5, lower right panel) and that for $461 \mathrm{DOS}=>\mathrm{RB}$ at the lower bath temperatures (top and middle panels of Figure 6), however, 462 suggests that with sufficient time, the liquid DOS (in the RB+DOS coagulated particles) wets the 463 RB surface and eventually completely encapsulates it, resulting in a core-shell-like structure 464 similar to that expected for $\mathrm{DOS}=>\mathrm{RB}$ but not possible for $\mathrm{RB}+\mathrm{SC}$ particles. This wetting also 465 results in increased physical, and thus thermal, contact between the two substances. RB contains 466 oxidized surfaces and is collapsed in water, and thus possesses the chemical and physical 467 properties favorable for wetting by DOS. Such a core-shell-like structure is consistent with the 468 results observed for the lagtime behavior of individual particles discussed above. Both RB+DOS 469 particles at the shortest mixing times and $\mathrm{RB}+\mathrm{SC}$ particles with positive lagtimes exhibit 470 scattering signals with a single maximum, similar to the example in Figure 4a, whereas the 471 scattering signals from RB+DOS particles at the longest mixing times exhibit two peaks, either 472 one before incandescence and one coincident with incandescence (Figure 4f), or one before 
473 incandescence and one after (Figure 4g). Scattering signals having two maxima are discussed 474 further below.

475 The DOS $=>\mathrm{RB}$ particles, in which the $\mathrm{rBC}$ is expected to be completely coated with DOS in a 476 core-shell-like configurations, and which thus have extensive thermal contact between the rBC 477 and non-rBC components, exhibit both positive and negative lagtimes (Figure 6). The fact that 478 the majority of such particles at the lower bath temperatures (i.e., those with thinner coatings) 479 exhibit positive lagtimes is likely due to the complete evaporation of the DOS before 480 incandescence of the $\mathrm{rBC}$. The trend of decreasing values of the positive lagtimes with 481 increasing $D_{\mathrm{me}, \mathrm{rBC}}$ can be explained by the fact that for a given DOS bath temperature, the 482 coating thickness is nearly the same for all particles, as noted above. With increasing $D_{\mathrm{me}, \mathrm{BBC}}$ 483 there is greater energy absorbed per mass of DOS coating, and thus this coating can be 484 evaporated more quickly, resulting in lower positive values of $\Delta \tau$. The existence of negative 485 lagtimes can be explained by some fraction of the particles having coatings so thick that the RB 486 is unable to transfer heat sufficiently rapidly, resulting in fragmentation, as observed in 487 laboratory settings by Moteki and Kondo (2007). The trend in negative lagtime values with 488 decreasing $D_{\mathrm{me}, \mathrm{rBC}}$, especially at the smallest particle sizes (lowest $D_{\mathrm{me}, \mathrm{rBC}}$ ), can be explained by 489 the increasing dissipation of heat to the gas via conduction, which will decrease the particle 490 heating rate, enabling more coating material to evaporate, thereby decreasing fragmentation.

491 Bimodal Scattering Distributions. As introduced above, the current operating definition of 492 lagtime utilizes only the scattering peak with the largest maximum. However, as a large fraction 493 of rBC-containing particles exhibit bimodal scattering signals, it is worth examining whether 494 these signals offer utility in studying rBC-containing morphology. To this end, distributions of $495 \Delta \tau_{2}$, defined as the difference between the time of the maximum of the incandescence signal and 496 that of the second maximum of the scattering signal, and $\Delta \tau_{1}$, the difference between the time of 497 the maximum of the incandescence signal and that of the first scattering maximum, are shown in 498 Figure 9. For a bimodal scattering signal, $\Delta \tau_{1}$ will always be greater than or equal to zero (as the 499 first scattering maximum will always occur before or simultaneous to incandescence), and the 500 lagtime will be equal to either $\Delta \tau_{1}$ or $\Delta \tau_{2}$, depending on which scattering maximum is larger.

501 The distributions of $\Delta \tau_{1}$ and $\Delta \tau_{2}$ for RB+SC and RB+DOS particles at the shortest $(<1 \mathrm{hr})$ 
502 mixing times are similar (as were the lagtime distributions for these particles), although the 503 distribution for the $\mathrm{RB}+\mathrm{DOS}$ particles contains one more cluster than that for $\mathrm{RB}+\mathrm{SC}$ particles.

504 Both contain a dominate cluster near $\Delta \tau_{1}=0.4 \mu$ s and $\Delta \tau_{2}=-6 \mu$ s and a secondary cluster near $505 \Delta \tau_{1}=7 \mu \mathrm{s}$ and $\Delta \tau_{2}=0.4 \mu \mathrm{s}$, but the distribution for RB+DOS particles has a less populated 506 cluster near the bisecting line $\left(\Delta \tau_{1}=-3 \mu \mathrm{s}\right.$ and $\left.\Delta \tau_{2}=-4 \mu \mathrm{s}\right)$, indicating that these bimodal 507 scattering signals are more nearly symmetric about the incandescence signal. In contrast, the 508 distributions for both types of particles at the longest mixing time each consist of only a single 509 cluster, but these are in different locations; that for $\mathrm{RB}+\mathrm{SC}$ is near the dominant cluster that 510 occurred at short mixing times for these particles, whereas that for RB+DOS particles is near the 511 less populated one for shorter mixing times that occurred near the bisecting line. The loss of the 512 cluster that was located near $\Delta \tau_{1}=\sim 7 \mu$ s and $\Delta \tau_{2}=0.4 \mu \mathrm{s}$ in both the RB+SC and RB+DOS

513 distributions at short times is ascribed to loss of particles through gravitational sedimentation, as 514 these particles were characterized by large RB components. This latter distribution is nearly 515 identical to that for $\mathrm{RB}=>\mathrm{DOS}$ particles (Figure 9, right column, lower panel), further supporting 516 the argument presented above that the morphologies of RB+DOS particles at the longest mixing 517 times and of DOS=>RB particles are similar.

518 Implications for ambient observations. The SP2 lagtime distribution observed by Sedlacek et al. 519 (2012) for an air mass containing aged biomass-burn tracers (Figure 4 of that publication), which 520 was obtained with the same SP2 instrument operating at the same conditions as the current 521 laboratory investigation (laser power $3000 \mathrm{~mA}$ and sample flow rate $120 \mathrm{~cm}^{3} \mathrm{~min}^{-1}$ ), is 522 qualitatively more similar to the second lagtime distribution pattern discussed above (i.e., for $523 \mathrm{RB}+\mathrm{DOS}$ particles at longer mixing times and for $\mathrm{DOS}=>\mathrm{RB}$ particles) than the first pattern (i.e., 524 for $\mathrm{RB}+\mathrm{SC}$ and $\mathrm{RB}+\mathrm{AS}$ particles and for $\mathrm{RB}+\mathrm{DOS}$ particles at the shorter two mixing times). 525 This is especially so for the dependence of the mode at negative lagtimes on $D_{\text {me,rBC }}$. The 526 biomass burning plume observed by Sedlacek et al. exhibited a lagtime distribution with three 527 prominent modes: one centered at large positive lagtimes at small values of $D_{\mathrm{me}, \mathrm{rBC}}$, one at small 528 positive lagtimes at larger values of $D_{\mathrm{me}, \mathrm{rBC}}$, and one at negative lagtimes with increasingly larger 529 negative values with increasing values of $D_{\mathrm{me}, \mathrm{BBC}}$. There was no evidence in the ambient biomass 530 burning observations of a negative lagtime mode comprised of small negative values of $\Delta \tau$ at 531 larger $D_{\text {me,rBC}}$, which has been found to dominate the lagtime distribution observed for the 
$532 \mathrm{RB}+\mathrm{SC}$ system. The background ambient observations reported by Sedlacek et al. (2012) always 533 exhibited measurable negative lagtimes, averaging $\sim 10 \%$ of the total number of rBC-containing 534 particles (not just those exclusive of the range of $\Delta \tau$ of 0.4 to $1.2 \mu \mathrm{s}$ ). Additionally, the 535 distribution of $\Delta \tau_{1}$ and $\Delta \tau_{2}$ for the ambient particles analyzed by Sedlacek et al. (2012) that have 536 two scattering signals, shown in Figure 9 (right column, upper panel), is similar to that for $537 \mathrm{RB}+\mathrm{DOS}$ particles at the longest mixing time and to that for the DOS $=>\mathrm{RB}$ particles, although it 538 extends over a wider range of values of $\Delta \tau_{2}$, possibly reflecting different values of $D_{\mathrm{me}, \mathrm{BBC}}$ and/or 539 different thermo-chemical properties for these particles.

540 The similarity between the lagtime distributions and the distributions of $\Delta \tau_{1}$ and $\Delta \tau_{2}$ for the 541 particles observed in the aged biomass burning plume and those of the laboratory RB+DOS and 542 DOS $=>$ RB particles suggests that the particles in the biomass burning plume had extensive 543 contact between the $\mathrm{rBC}$ and non-rBC components. There are several morphologies that would 544 allow for this. For rBC that has been collapsed into a nearly spherical shape (as in the current 545 laboratory investigation), one such morphology is complete encapsulation by the non-rBC 546 substance, as would be expected for the DOS $=>$ RB particles-whether in a core-shell or offcenter (eccentric) configurations. However, if the $\mathrm{rBC}$ retained a fractal-like structure, as is 548 typical for nascent soot in the atmosphere, then a morphology that would allow for extensive 549 thermal contact is one in which the rBC is on the surface (as suggested by Sedlacek et al.) and is 550 similar to an octopus (as a surrogate for the fractal-like nascent soot) holding onto a rock (as a 551 surrogate for the non-rBC substance) with its tentacles. The current results further suggest that 552 the differences between the ambient background particles and the biomass burning particles 553 observed by Sedlacek et al. were due to different morphologies and/or different thermo-chemical 554 properties of the non-rBC material. Although the specific morphology of these particles cannot 555 be positively identified using the SP2 lagtime approach, the observations may provide useful 556 information on source attribution and population-based mixing state for biomass particles.

557 As discussed earlier, Moteki and co-workers (2014) have developed a mixing-state analysis 558 methodology based on the behavior of time-dependent scattering cross section at the onset of $559 \mathrm{rBC}$ incandescence. Our results suggest that the operating conditions of the SP2 (laser power 560 and sample flow rate), the thermochemical properties of the non-rBC material, and the contact 561 between the non-rBC and $\mathrm{rBC}$ will affect the temporal behavior of the scattering cross-section 
562 between non-refractory sublimation and $\mathrm{rBC}$ incandescence, the timeframe considered by

563 Moteki et al. For example, a lower heating rate imposed on a coagulated $\mathrm{rBC}$ particle - as would 564 occur if the sample flow rate were reduced - would increase the likelihood of sublimation of the 565 non-refractory material. This could lead to a condition that more closely mimics the observed 566 behavior ascribed to core-shell configurations by exhibiting material evaporation well before $567 \mathrm{rBC}$ incandescence.

568 In situations with high rBC particle number concentrations, such as biomass burning plumes, 569 reduction of the sample flow rate to minimize the effects of coincidence counting will decrease 570 particle heating rate and will likely change the response of the rBC-containing particle in the SP2.

571 Future analysis using the present data set will specifically examine the effects of SP2 operating 572 conditions on the Moteki mixing state analysis in an effort to better map out the performance 573 envelope of this analysis methodology.

\section{Conclusions}

575 This study demonstrates and highlights several important aspects related to the behavior of rBCcontaining particles in the SP2, specifically particle fragmentation, which is important for

577 lagtime analyses and may also be important for other analyses: (1) SP2 operating conditions 578 affect the frequency of particle fragmentation; (2) the physical and chemical properties of rBC579 containing particles affect the frequency of fragmentation; and (3) the SP2 appears to fragment a 580 substantial fraction of rBC-containing particles under most operating conditions.

581 At this point it is worth examining the utility of lagtimes and lagtime distributions for making 582 inferences about the morphologies and thermo-chemical properties of $\mathrm{rBC}$-containing particles. 583 From our results, we offer the following observations. First, two different general lagtime 584 distribution patterns were observed in these experiments: one resulting from coagulation of RB 585 with either solid SC or solid AS, or with liquid DOS at short mixing times, and the other resulting from condensation of liquid DOS onto RB or from coagulation of RB with liquid DOS 587 particles at longer mixing times. These different lagtime distributions are attributed to different 588 particle morphologies. The dependence of the lagtime distribution of coagulated RB+DOS 589 particles on mixing time can be explained by wetting of the RB by the DOS, with the lagtime 

distribution (and the morphology of the particles) changing from being similar to that for $\mathrm{RB}+\mathrm{SC}$ particles to becoming more similar to that for $\mathrm{DOS}=>\mathrm{RB}$ particles.

592 Second, this study demonstrates the dominant role played by SP2 operating conditions, 593 specifically laser power and sample flow rate, in determining the observed lagtime distributions. 594 These operating conditions influence the rates of laser heat uptake and internal particle heat 595 transfer (and dissipation) within rBC-containing particles, which in turn affect the fraction of 596 particles that fragment in the SP2 laser and generate negative lagtimes in particular, and the 597 observed lagtime distributions in general. The effects of operating conditions are shown 598 explicitly for coagulated $\mathrm{RB}+\mathrm{SC}$ and $\mathrm{RB}+\mathrm{AS}$ particles and for $\mathrm{DOS}=>\mathrm{RB}$ particles. In all cases, 599 the positive-to-negative lagtime ratio is shown to be dependent upon these conditions: lower 600 (higher) laser power and lower (higher) sample flow rate generates slower (faster) particle 601 heating and higher (lower) positive-to-negative lagtime ratios. Comparison of lagtime results 602 between different studies with different SP2 instrument parameters will therefore be challenging 603 at best, and to enable meaningful comparisons and to further analyses of SP2 measurements, it is 604 imperative that SP2 laser power and sample flow rate be reported for all future studies. The best 605 practice for quantifying laser power is to measure the scattered light from a standard particle 606 (Schwarz et al., 2010), and the best practice for quantifying particle velocities is to measure the 607 transit times (e.g., FWHM) for non-absorbing particles passing through the laser beam (Schwarz 608 et al., 2015), both of which can be readily accomplished using the same standard particles (e.g., $609220 \mathrm{~nm}$ PSL).

610 Third, for given SP2 operating conditions, lagtime distributions are also dependent on the 611 thermo-chemical properties of the non-rBC substance. Comparison of lagtime distributions for 612 coagulated $\mathrm{RB}+\mathrm{SC}$ and $\mathrm{RB}+\mathrm{AS}$ particles reveals measurable differences in behavior (e.g., $613 \mathrm{RB}+\mathrm{AS}$ particles consistently generated higher positive-to-negative lagtime ratios), which are 614 attributed to the greater thermal volatility of AS than that of SC.

615 Fourth, all particle morphologies and compositions examined here, whether the rBC was on the 616 surface (e.g., RB+SC) or inside the particle (e.g., DOS=>RB), exhibited both positive and 617 negative lagtimes. Such a result can confound lagtime-based mixing-state analyses, even those 618 involving examination of the individual particle signals. As positive lagtimes from particles with 
$619 \mathrm{rBC}$ on the surface can mimic those from particles with core-shell configurations in all aspects, it 620 is not possible to attribute positive lagtimes to a core-shell configuration. Such an attribution 621 could lead to erroneous conclusions, including those regarding radiative properties (especially 622 absorption). The concept of coating thickness for such particles, as is typically obtained from 623 signal scattering and incandescence amplitudes with the implicit assumption of a core-shell 624 configuration, would (of course) be meaningless.

625 Fifth, the mere presence of a negative lagtime cannot be used to infer particle morphology, as 626 both solid-solid particles and core-shell particles with thick coatings can exhibit negative 627 lagtimes. In this regard, the conclusions of Dahlkötter et al. (2014) that negative lagtimes result 628 only from thickly-coated particles in a core-shell configuration is not supported by our 629 observations. The SP2 technique appears to fragment a substantial fraction of rBC-containing 630 particles under most operating conditions, both under ambient conditions and in laboratory 631 experiments.

632 Sixth, although the current results indicate that SP2 lagtime analysis is not able to 633 unambiguously determine particle morphology or to provide information on the thermo-chemical 634 properties of the non-rBC component directly, the lagtime distribution observations may provide 635 useful information on source attribution and population-based mixing states for rBC-particle 636 populations with different lagtime characteristics. For example, the observations reported by 637 Sedlacek et al. (2012) of two types of rBC-containing particles defined by their lagtime 638 differences, using the same instrument with constant operating conditions, were highly correlated 639 with ambient background particles and tracers of biomass burning transported over the sampling 640 site. Additionally, the strong dependence of the magnitudes and signs of lagtimes on SP2 641 operating conditions might be exploited to probe $\mathrm{rBC}$-containing particles for additional 642 information. For example, the laser power could be modulated for times that would be 643 sufficiently long to yield good statistics, but sufficiently short that properties of the aerosol 644 sampled would remain constant.

645 Finally, both positive and negative lagtimes may derive from bimodal light scattering signals 646 (Figure 4c-f), a subtlety not appreciated by the current definition of lagtime. The lagtime 647 methodology reduces the measured scattering and incandescence signals for each rBC-containing 
648 particle to a single quantity, which cannot account for bimodal scattering signals nor can it 649 unambiguously discriminate between effects due to SP2 operating conditions or varying rBC

650 chemical and physical properties. Likewise, the two measured quantities employed by Moteki 651 et al. (2014) would also be expected to be sensitive to the operating conditions. Future work will 652 investigate the full scattering and incandescence signals of individual particles (e.g., Figure 9) 653 and their sensitivities to details of particle morphology and thermo-chemical properties with the 654 goal of identifying meaningful quantities that are insensitive to the SP2 operating conditions.

\section{Acknowledgements}

656 This research was performed under sponsorship of the U.S. DOE Office of Biological \& 657 Environmental Sciences (OBER) Atmospheric Research Program (ASR) under contracts DE658 AC02-98-CH10886 (BNL), DE-SC0006980 (Boston College), DE-FG02-05ER63995 (ARI) 659 NSF Award Numbers. ATM-0854916 and 1244918 (Boston College), EPA STAR grant No. 66083503301 (Boston College). Researchers thank the DOE Atmospheric Radiation Measurement 661 (ARM) Climate Research Facility for use of the Single Particle Soot Photometer (SP2). 


\section{References}

664 Bond, T. C., plus 30-authors (2013) Bounding the role of black carbon in the climate system: A scientific assessment, J. Geophys. Res., 118, doi:10.1002/jgrd.50171.

666

667

668

669

670

671

672

673

674

675

676

677

678

679

680

681

682

683

684

685

686

Bond, T. C., and R. W. Bergstrom (2006), Light absorption by carbonaceous particles: An investigative review, Aerosol Sci. Technol., 40, 27-67, doi:10.1080/02786820500421521.

Bond, T. C., G. Habib, and R. W. Bergstrom (2006), Limitations in the enhancement of visible light absorption due to mixing state, J. Geophys. Res., 111: 1-13.

Cappa, C. D., T. B. Onasch, P. Massoli, D. R. Worsnop, T. S. Bates, E. S. Cross, P. Davidovits, J. Hakala, K. L. Hayden, B. T. Jobson, K. R. Kolesar, D. A. Lack, B. M. Lerner, S.-M. Li, D. Mellon, I. Nuaaman, J. S. Olfert, T. Petäjä, P. K. Quinn, C. Song, R. Subramanian, E. J. Williams, and R. A. Zaveri (2012), Radiative absorption enhancements due to the mixing state of atmospheric black carbon, Science, 337, 1078-1081, doi:10.1126/science.1223447.

Conant, W. C., Nenes, A., and Seinfeld J. H., (2002) Black carbon radiative heating effects on cloud microphysics and implications for the aerosol indirect effect: 1. Extended Kohler theory, J. Geophys. Res., 107, D21, 4604, doi:10.1029/2002JD002094, 2002.

Dahlkötter, F., M. Gysel, D. Sauer, A. Minikin, R. Baumann, P. Seifert, A. Ansmann, M. Fromm, C. Voigt, and B. Weinzierl (2013), The Pagami Creek smoke plume after long-range transport to the upper troposphere over Europe-aerosol properties and black carbon mixing state, Atmos. Chem. Phys.., 14, 6111-6137, 2014, doi:10.5194/acp-14-6111-2014.

Fuller, K. A., W. C. Malm, and S. M. Kreidenweis (1999), Effects of mixing on extinction by carbonaceous particles, J. Geophys. Res., 104, D13, 15941-15954.

Fuller, K. A. (1995a), Scattering and absorption cross sections of compounded spheres. III. Spheres containing arbitrarily located spherical inhomogeneities, J. Opt. Soc. Amer. A, 12, 893-904. 
Fuller, K. A. (1995b), Scattering and absorption cross sections of compounded spheres. II. Calculations for external aggregation, J. Opt. Soc. Amer. A, 12, 881-892.

Gao, R. S., J. P. Schwartz, K. K. Kelly, D. W. Fahey, L. A. Watts, T. L. Thompson, J. R. Spackman, J. G. Slowik, E. S. Cross, J.-H. Han, P. Davidovits, T. B. Onasch, and D. R. Worsnop (2007), A novel method for estimating light-scattering properties of soot aerosols using a modified Single-Particle Soot Photometer, Aerosol Sci. Technol., 41, 125-135, doi:10.1080/02786820601118398.

Jacobson, M. Z. (2000), A physically-based treatment of elemental carbon optics: Implications for global direct forcing of aerosols, Geophys. Res. Lett., 27, 217-220.

Jacobson, M. Z. (2001), Strong radiative heating due to the mixing state of black carbon in atmospheric aerosols, Nature, 409, 695-697.

Koch, D., and Del Genio, A. D., (2010) Black carbon semi-direct effects on cloud cover: review and synthesis, Atmos. Chem. Phys., 10, 7685-7696, doi:10.5194/acp-10-7685-2010.

Lack, D.A., Cappa, C.D., Cross, E.S., Massoli, P., Ahern, A.T., Davidovits, P., and Onasch, T.B. (2009), Absorption enhancement of coated absorbing aerosols: Validation of the photoacoustic technique for measuring the enhancement, Aerosol Sci. Technol., 43, 1006-1012, doi:10.1080/02786820903117932.

Moteki, N., and Y. Kondo (2007), Effects of mixing state on black carbon measurements by laser-induced incandescence, Aerosol Sci. Technol., 41:4, 398-417, doi:10.1080/02786820701199728.

Moteki N., and Y. Kondo (2008), Method to measure time-dependent scattering cross-sections of particles evaporating in a laser beam, J. Aerosol Sci., 39, 348-364, doi:10.1029/2006JD007076.

Moteki, N., Y. Kondo, and K. Adachi (2014), Identification by single-particle soot photometer of black carbon particles attached to other particles: Laboratory and ground observations in Tokyo, J. Geophys. Res., 119, 1031-1043, doi:10.1002/2013JD020655. 
O'Hanlon, J. F. (2003), A User's Guide to Vacuum Technology, 3rd Edition, John Wiley and Sons, Inc., $516 \mathrm{pp}$.

Onasch, T., B., Trimborn, A., Fortner, E. C., Jayne, J. T., Kok, G. L., Williams, L. R., Davidovits, P., and Worsnop D. R. (2012), Soot particle aerosol mass spectrometer: Development, validation, and initial application. Aerosol Sci. Technol., 46, 804-817, doi:10.1080/02786826.2012.663948.

Petzold, A., J. A. Ogren, M. Fiebig, P. Laj, S.-M. Li, U. Baltensperger, T. Holzer-Popp, S. Kinne, G. Pappalardo, N. Sugimoto, C. Wehrli, A. Wiedensohler, and X.-Y. Zhang (2013), Recommendations for reporting "black carbon" measurements, Atmos. Chem. Phys., 13, 8365-8379, doi:10.5194/acp-13-8365-2013.

Prather, K. A., T. Nordmeyer, and K. Salt (1994), Real-time characterization of individual aerosol particles using Time-of-Flight Mass Spectrometry, Anal. Chem., 66, 1403-1407.

Ramanathan, V., and G. Carmichael (2008), Global and regional climate changes due to black carbon, Nature Geosci., 1, 221-227.

Schwarz, J. P., R. S. Goa, D. W. Fahey, D. S. Thomson, L. A. Watts, J. C. Wilson, J. M. Reeves, M. Darbeheshti, D. G. Baumgardner, G. L. Kok, S. H. Chung, M. Schulz, J. Hendricks, A. Lauer, B. Kärcher, J. G. Slowik, K. H. Rosenlof, T. L. Thompson, A. O. Langford, M. Loewenstein, and K. C. Aikin (2006), Single-particle measurements of midlatitude black carbon and light-scattering aerosols from the boundary layer to the lower stratosphere. $J$. Geophys. Res., 111, D16207, doi:10.1029/2006JD007076.

Schwarz, J. P., J. R. Spackman, D. W. Fahey, R. S. Gao, U. Lohmann, P. Stier, L. A. Watts, D. S. Thomson, D. A. Lack, L. Pfister, M. J. Mahoney, D. Baumgardner, J. C. Wilson, and J. M. Reeves (2008), Coatings and their enhancement of black carbon light absorption in the tropical atmosphere, J. Geophys. Res., 113, D03203, doi:10.1029/2007JD009042.

Schwarz, J. P., J. R. Spackman, R. S. Gao, A. E. Perring, E. Cross, T. B. Onasch, A. Ahern, W. Wrobel, P. Davidovits, J. Olfert, M. K. Dubey, C. Massoleni, and D. W. Fahey (2010), The 
detection efficiency of the Single Particle Soot Photometer, Aerosol Sci. Technol., 44, 612628, doi:10.1080/02786826.2010.481298.

741 Schwarz, J. P., A. E. Perring, M. Z. Markovic, R. S. Gao, S. Ohata, J. Langridge, D. Law, R.

742 McLaughlin, R. and D. W. Fahey (2015) Technique and theoretical approach for quantifying the hygroscopicity of black-carbon-containing aerosol using a single particle soot photometer, J. Aerosol Sci., 81, 110-126, doi:10.1016/j.jaerosci.2014.11.009.

Sedlacek, A. J., III, E. R. Lewis, L. Kleinman, J. Xu, and Q. Zhang (2012), Determination of and evidence for non-core-shell structure of particles containing black carbon using the SingleParticle Soot Photometer (SP2), Geophys. Res. Lett., 39, doi:10.1029/2012GL050905.

Subramanian, R., G. L. Kok, D. Baumgardner, A. Clarke, Y. Shinozuka, T. L. Campos, C. G. Heizer, B. B. Stephens, B. de Foy, P. B. Voss, and R. A. Zaveri (2010), Black carbon over Mexico: the effect of atmospheric transport on mixing state, mass absorption cross-section, and BC/CO ratios, Atmos. Chem. Phys., 10, 219-237, doi:10/5194/acp-10-209-2010.

Tang, I. N. (1996), Chemical and size effects of hygroscopic aerosols on light scattering coefficients, J. Geophys. Res., 101, D14, 19245-19250.

754 Zaveri, R., J. C. Barnard, R. C. Easter, N. Riemer, and M. West (2010), Particle-resolved 755 simulation of aerosol size, composition, mixing state, and the associated optical and cloud 756 condensation nuclei activation properties in an evolving urban plume, J. Geophys. Res., 115, 757 D17210, doi:10.1029/2009JD013616. 
761 Figure 1: Experimental setup used to coagulate Regal black (RB) with sodium chloride (SC),

762 ammonium sulfate (AS), and dioctyl sebacate (DOS). Not shown is the coating chamber used for $763 \mathrm{RB}=>\mathrm{DOS}$ coating experiments.

765 Figure 2: Size distributions of Regal black (RB) number concentration, $d N_{\mathrm{BC}} / d \log D_{\mathrm{me}, \mathrm{rBC}}$, in 766 terms of mass-effective diameter of RB, $D_{\mathrm{me}, \mathrm{rBC}}$, measured by the Single Particle Soot

767 Photometer (SP2) for different values of mixing time $\left(t_{\mathrm{mix}}\right)$.

769 Figure 3: The inverse of the full width at half maximum (FWHM) for scattering signal from 770 individual $\mathrm{NaCl}$ particles as a function of the sample flow rate. Each data point is derived from $771 \sim 15,000$ individual particles.

773 Figure 4: Examples of scattering and incandescence signals from individual RB+SC (a-e) and 774 RB+DOS (f-g) particles: a) scattering signal maximum occurs before incandescence signal 775 maximum; b) scattering signal maximum coincides with incandescence signal maximum; c) 776 scattering signal maximum occurs after incandescence signal maximum; d) bimodal scattering 777 signal with scattering signal maximum coincident with incandescence signal maximum and a 778 second scattering peak after incandescence; e) bimodal scattering signal with scattering signal 779 maximum occurring after incandescence and a second scattering peak before incandescence; f) 780 bimodal scattering signal with scattering signal maximum coincident with incandescence signal 781 maximum and a second scattering peak before incandescence; and g) bimodal scattering signal 782 with scattering signal maxima roughly equal in magnitude, but opposite distance from the 783 incandescence signal maximum. 
Figure 5: Lagtime distributions for $\mathrm{RB}+\mathrm{SC}$ particles (left column), $\mathrm{RB}+\mathrm{AS}$ particles (center

786 column), and RB+DOS particles (right column) at different mixing times, determined by binning

787 events in ranges of lagtime, $\Delta \tau(400 \mathrm{~ns})$ and $\mathrm{rBC}$ mass-equivalent diameter, $D_{\mathrm{me}, \mathrm{rBC}}(5 \mathrm{~nm})$.

788 Colors represent the number fraction of particles within given ranges of $\Delta \tau$ and $D_{\text {me,rBC. As the }}$ 789 majority of RB particles remain uncoagulated, lagtimes of these particles are not included in the

790 figures. Also shown are the normalized size distributions of RB-number concentration,

$791 d N_{\mathrm{rBC}} / d \log D_{\mathrm{me}, \mathrm{rBC}}$, and the positive-to-negative lagtime ratio (number of particles with positive

792 lagtimes to those with negative lagtimes).

794 Figure 6: Lagtime distributions for DOS $=>$ RB condensation particles at different DOS bath 795 temperatures (middle column) and operating conditions, i.e., sample flow rate and injection laser 796 current, which controls laser power (bottom row). RB-number concentration, $d N_{\mathrm{rBC}} / d \log D_{\mathrm{me}, \mathrm{rBC}}$, 797 the same for all experiments, is shown only in the top central panel. Positive-to-negative lagtime ratios (number of particles with positive lagtimes to those with negative lagtimes) are also shown.

Figure 7: Dependence of RB+SC lagtime distributions on SP2 operating conditions, i.e., sample flow rate (increasing left to right) and injection laser current, which controls laser power (top

802 row: $2000 \mathrm{~mA}$, bottom row: $3000 \mathrm{~mA}$ ). Also shown are the normalized size distributions of RB803 number concentration, $d N_{\mathrm{rBC}} / d \log D_{\mathrm{me}, \mathrm{rBC}}$, and the positive-to-negative lagtime ratio (number of 804 particles with positive lagtimes to those with negative lagtimes).

Figure 8: Dependence of positive-to-negative lagtime ratio (number of particles with positive

807 lagtimes to those with negative lagtimes) on SP2 operating conditions, i.e., sample flow rate and 808 injection laser current, which controls laser power.

810 Figure 9: Distributions of $\Delta \tau_{1}$ (the difference between the time of the maximum of the 811 incandescence signal and that of the first maximum of the scattering signal) and $\Delta \tau_{2}$ (the 
812 difference between the time of the maximum of the incandescence signal and that of the second 813 scattering maximum) for bimodal scattering signals from RB+SC (left column) and RB+DOS

814 (center column) particles, at the shortest (top panels in each column) and longest (bottom panels)

815 mixing times. Right column contains distributions for ambient particles from Sedlacek et al.

816 (2012) (top panel) and from bimodal scattering distributions for RB $=>$ DOS particles (lower

817 panel). Color represents number fraction of particles in given ranges of $\Delta \tau_{1}$ and $\Delta \tau_{2}$. Dotted line

818 bisecting the lower right quadrant of each plot denotes where the first and second scattering

819 peaks are equidistant (temporally) from the incandescence peak. 
821 Figure 1

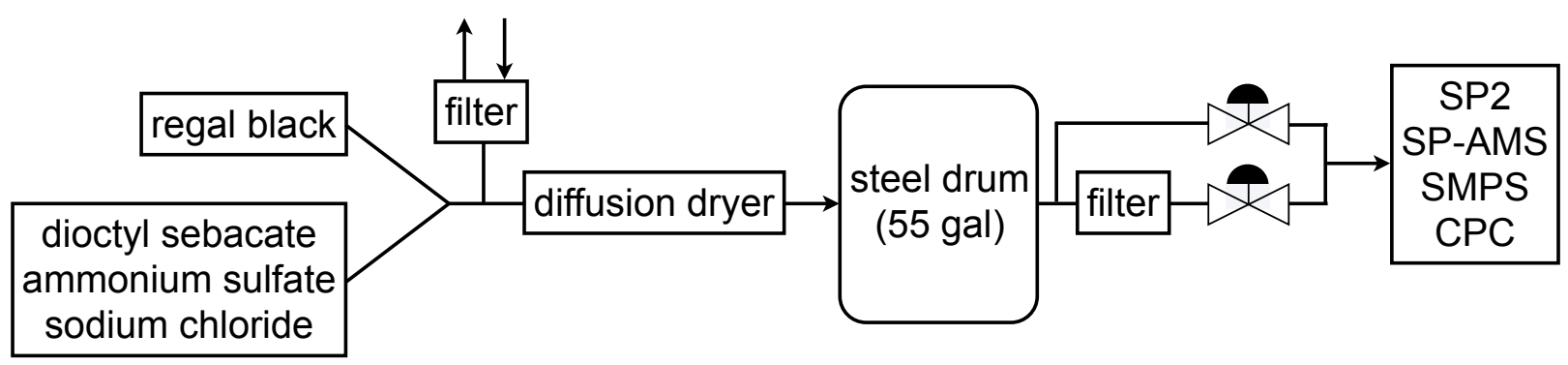

823 
$824 \quad$ Figure 2

825

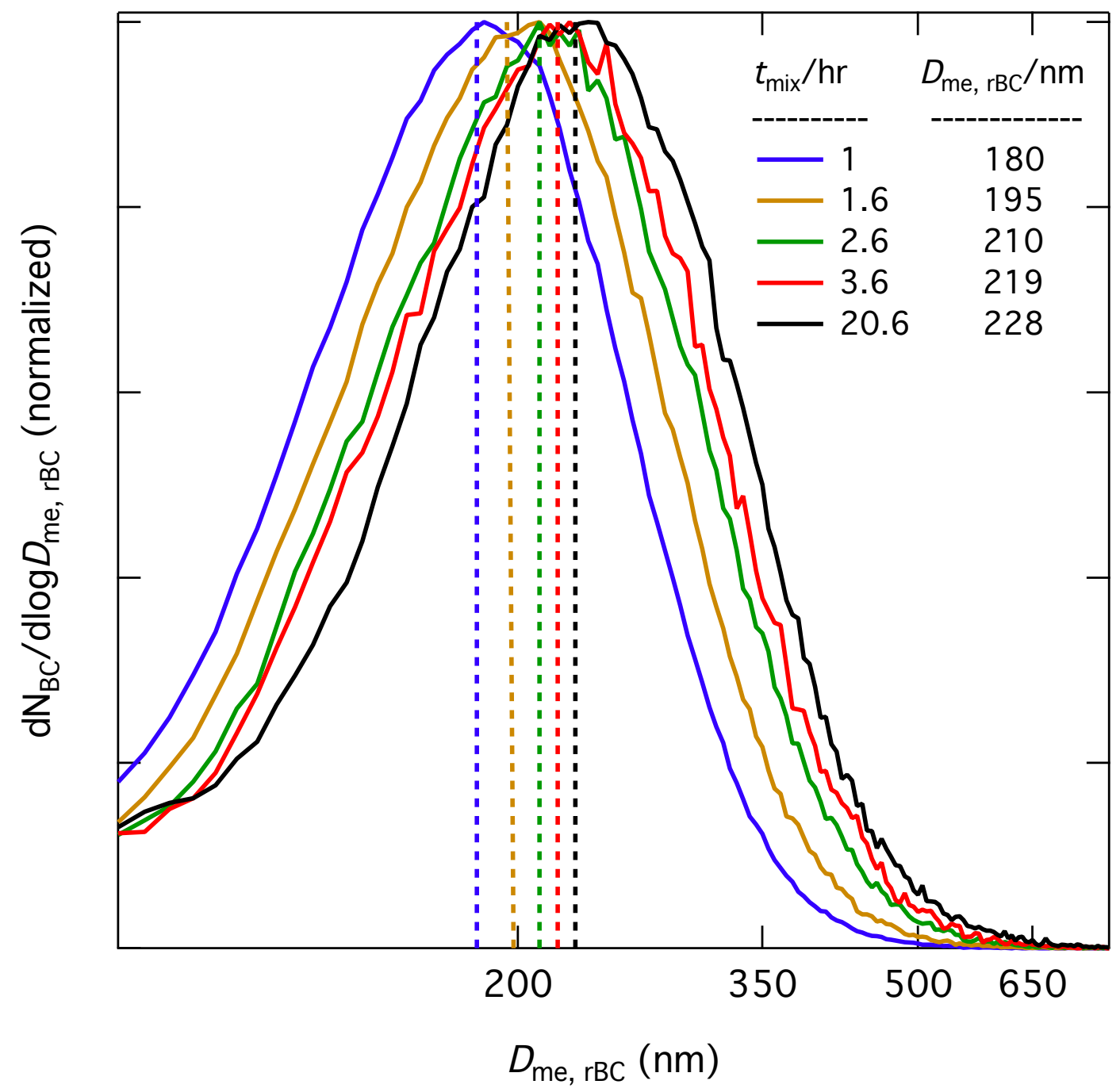

826

827 
$828 \quad$ Figure 3

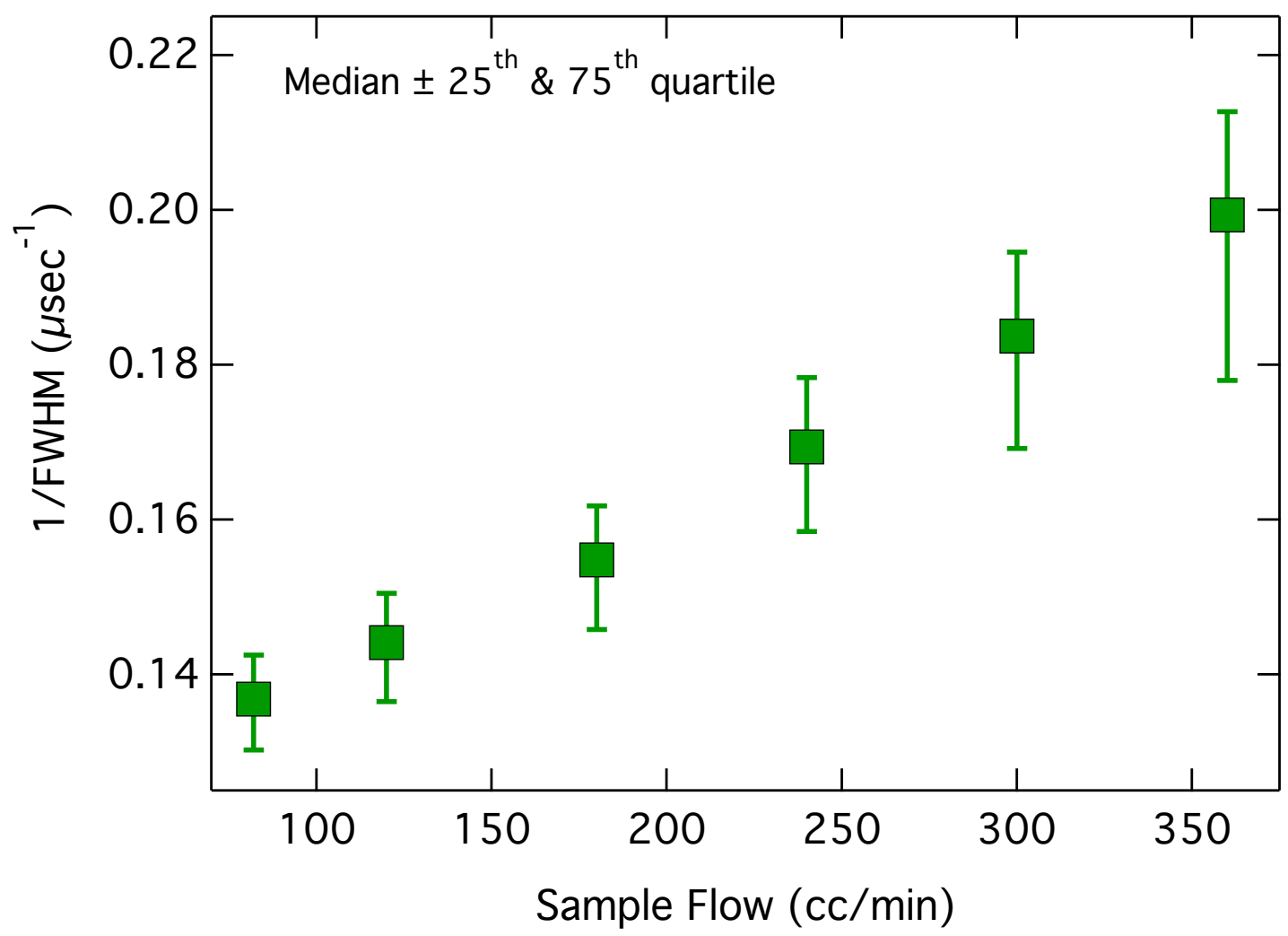

829 


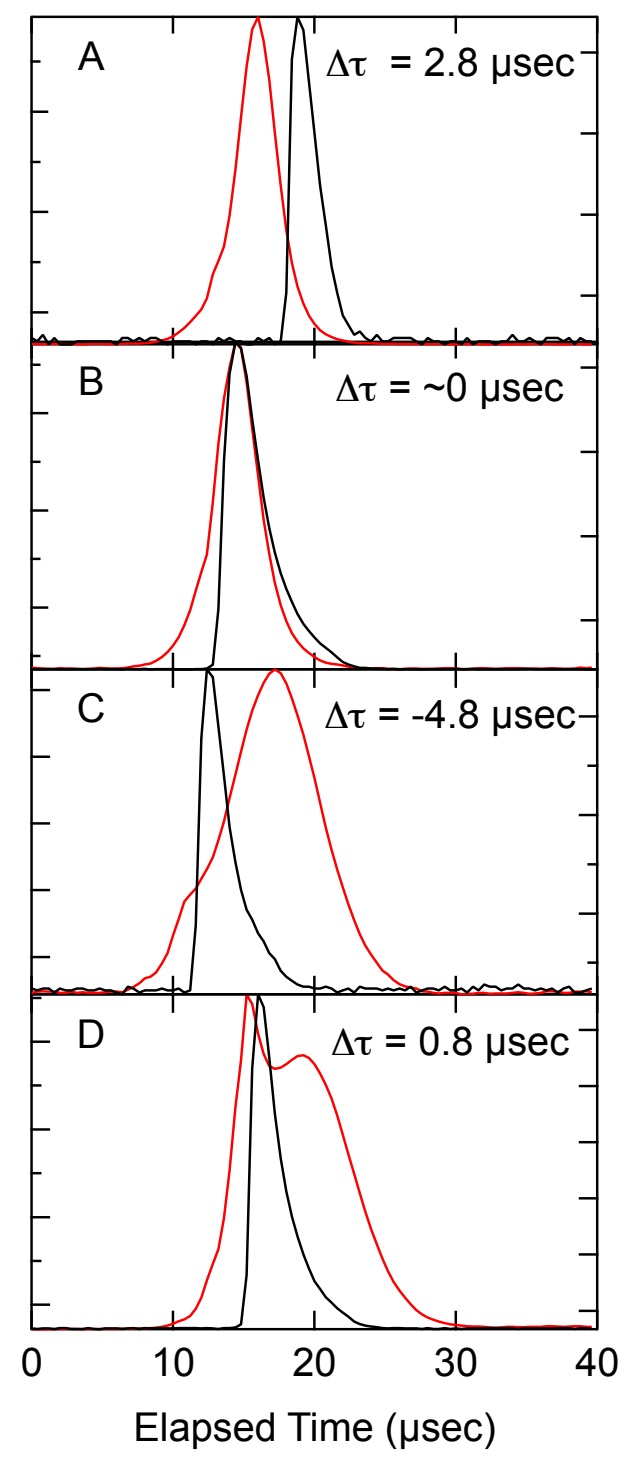

832

833

834

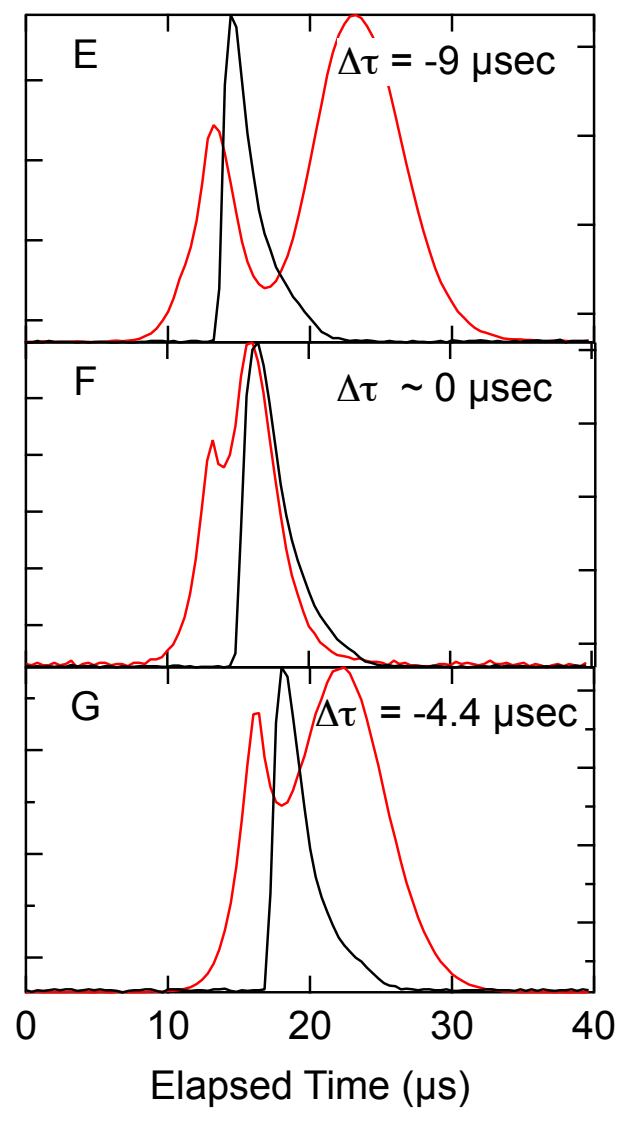

Scattering Incandescence 
Figure 5

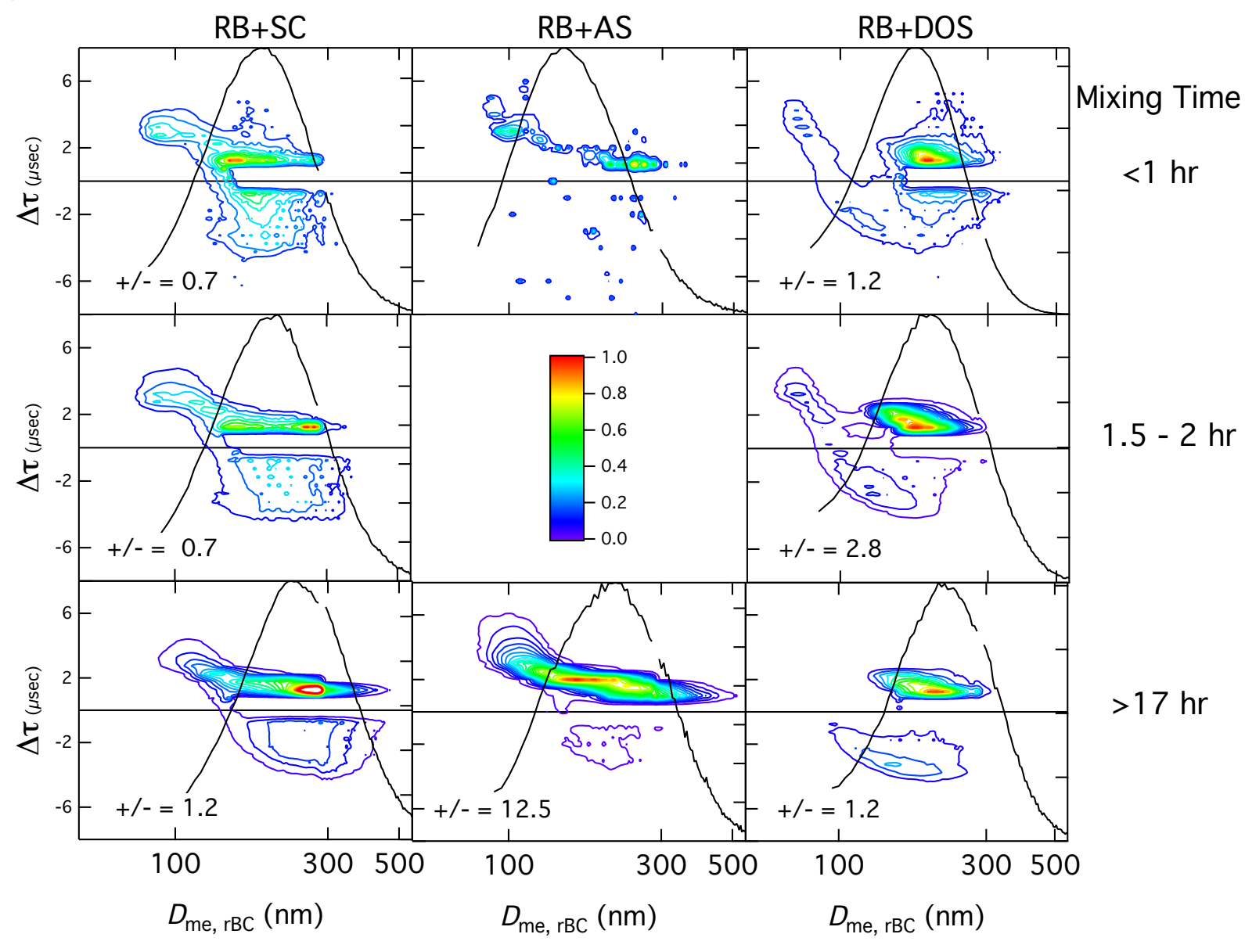




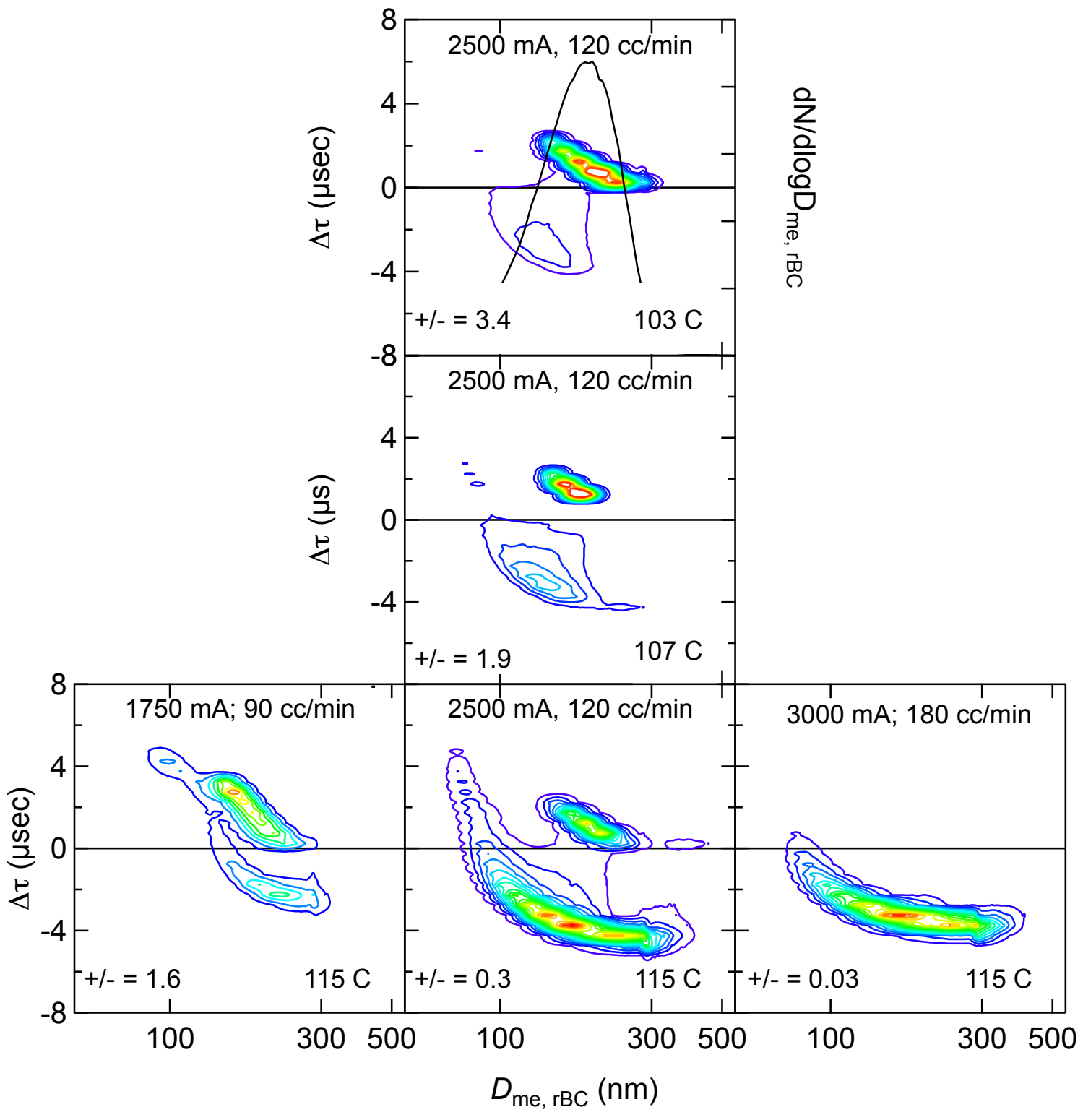




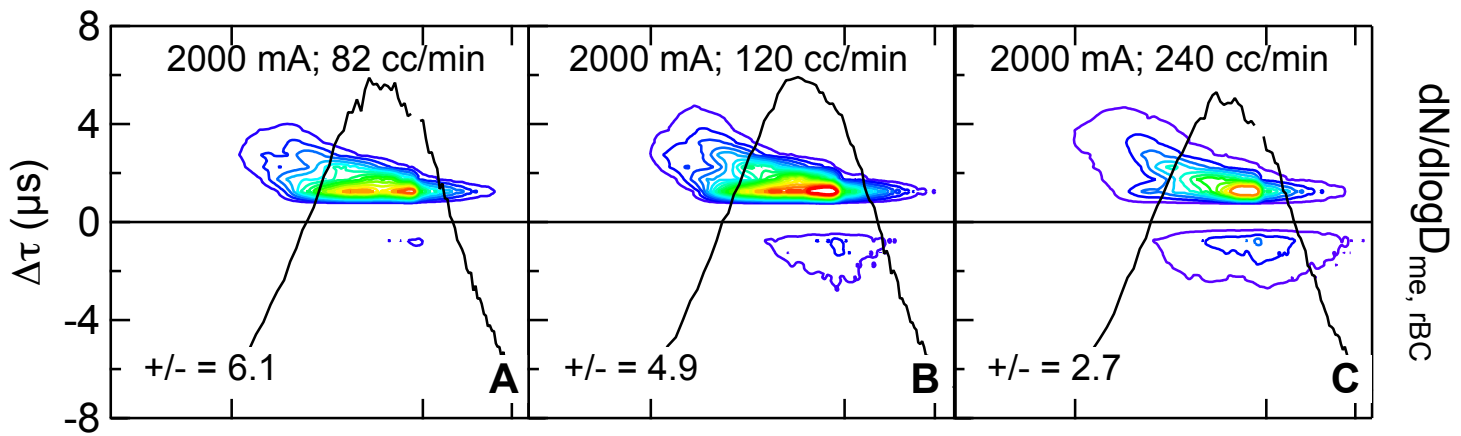

846

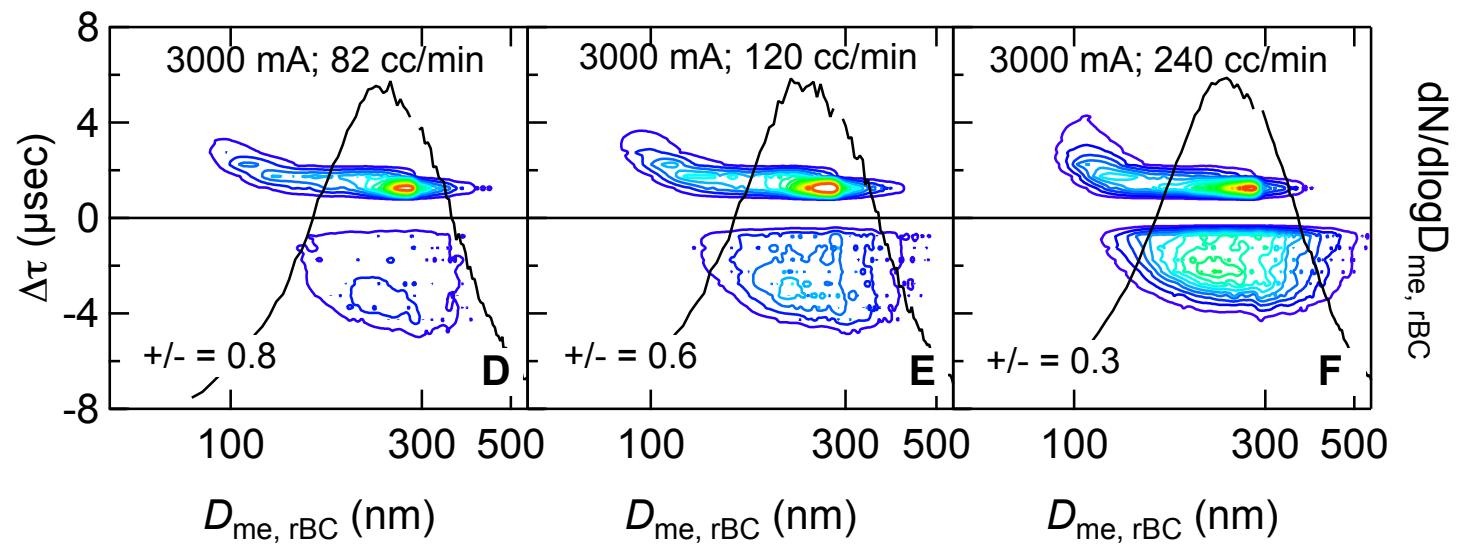

847

848 
$849 \quad$ Figure 8

850

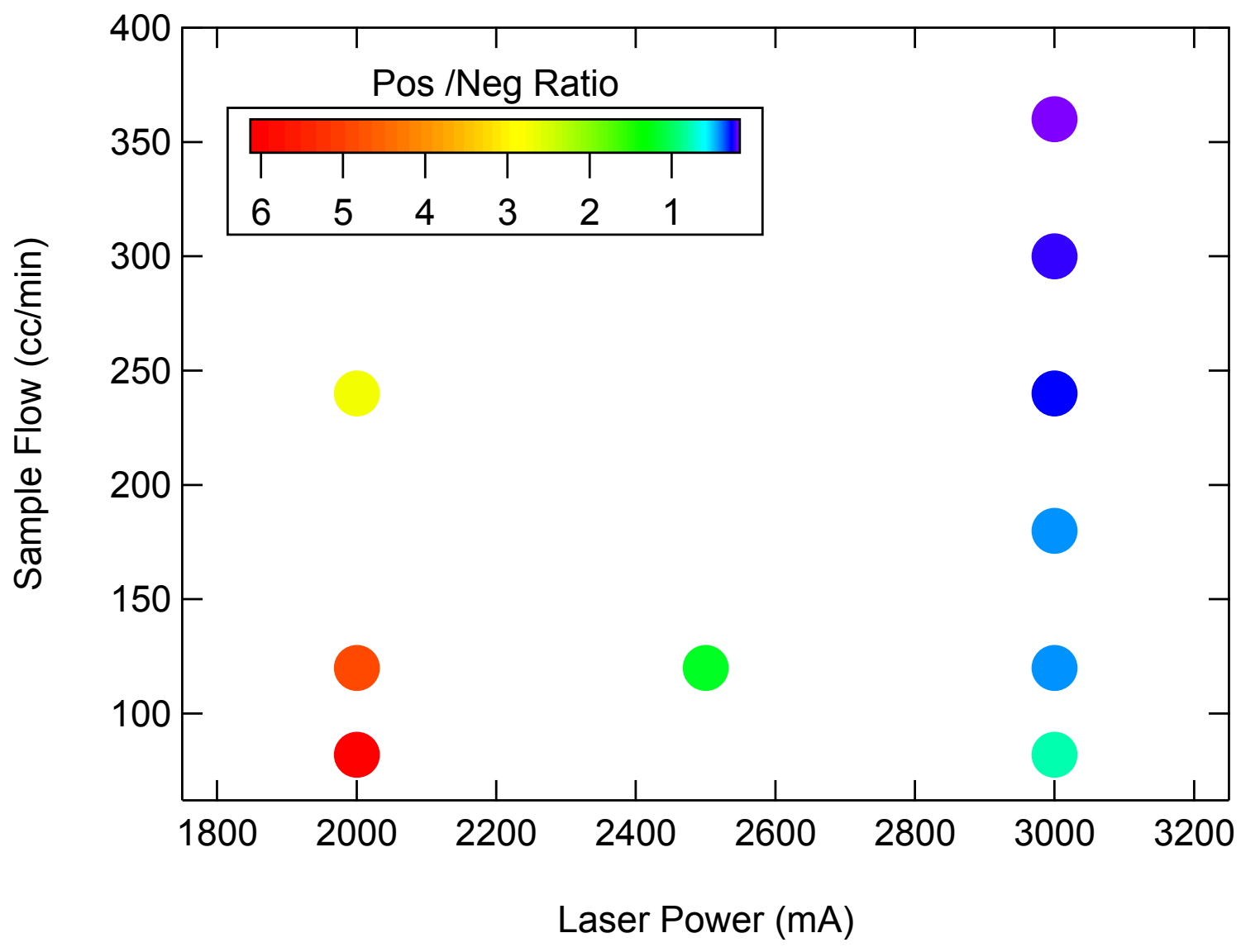

851

852

853 


\section{Figure 9}

855
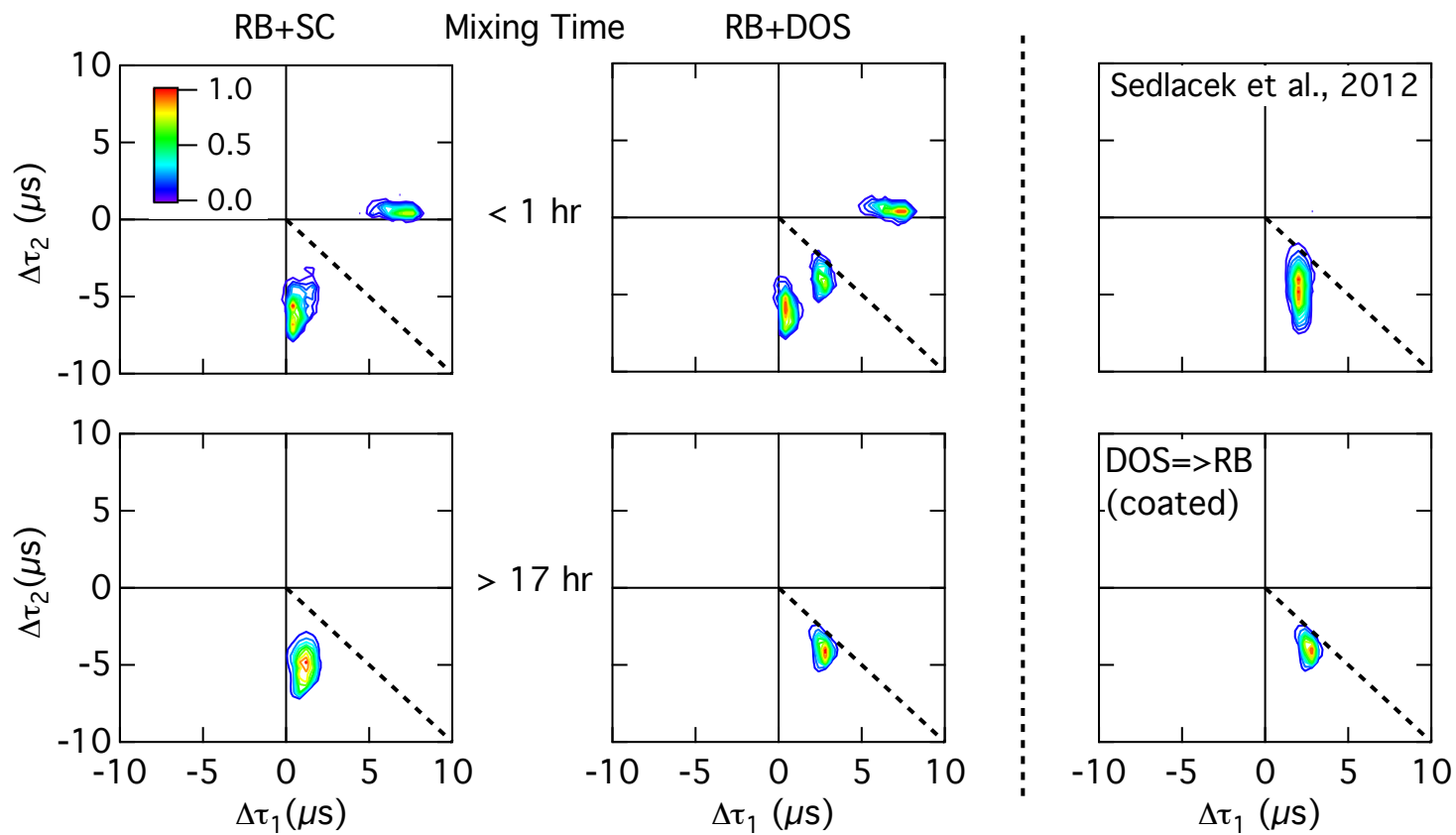

856 